\title{
Scalable and High-Fidelity Quantum Random Access Memory in Spin-Photon Networks
}

\author{
K. C. Chen $\odot,{ }^{1,2}$ W. Dai $\odot,{ }^{1,3}$ C. Errando-Herranz $\odot,{ }^{1}$ S. Lloyd,,${ }^{1,4}$ and D. Englund $\circledast^{1,2, *}$ \\ ${ }^{1}$ Research Laboratory of Electronics, Massachusetts Institute of Technology, Cambridge, Massachusetts 02139, \\ USA \\ ${ }^{2}$ Department of Electrical Engineering and Computer Science, Massachusetts Institute of Technology, Cambridge, \\ Massachusetts 02139, USA \\ ${ }^{3}$ Department of Computer Science, University of Massachusetts, Amherst, Massachusetts 01003, USA \\ ${ }^{4}$ Department of Mechanical Engineering, Massachusetts Institute of Technology, Cambridge, Massachusetts \\ 02139, USA
}

(Received 16 March 2021; revised 11 June 2021; accepted 6 July 2021; published 3 August 2021)

\begin{abstract}
A quantum random access memory (qRAM) is considered an essential computing unit to enable polynomial speedups in quantum information processing. Proposed implementations include the use of neutral atoms and superconducting circuits to construct a binary tree but these systems still require demonstrations of the elementary components. Here, we propose a photonic-integrated-circuit (PIC) architecture integrated with solid-state memories as a viable platform for constructing a qRAM. We also present an alternative scheme based on quantum teleportation and extend it to the context of quantum networks. Both implementations realize the two key qRAM operations, (1) quantum state transfer and (2) quantum routing, with already demonstrated components: electro-optic modulators, a Mach-Zehnder interferometer (MZI) network, and nanocavities coupled to artificial atoms for spin-based memory writing and retrieval. Our approaches furthermore benefit from built-in error detection based on photon heralding. Detailed theoretical analysis of the qRAM efficiency and query fidelity shows that our proposal presents viable near-term designs for a general qRAM.
\end{abstract}

DOI: 10.1103/PRXQuantum.2.030319

\section{INTRODUCTION}

Random access memory (RAM) is a fundamental computing unit that allows on-demand storage and retrieval of data. While a classical RAM addresses one memory cell in the database per operation, a quantum RAM (qRAM) permits the querying of a superposition of multiple memories [1]. Given a superposition of addresses $j$, the "qRAM" returns a correlated set of data $D_{j}$ :

$$
|\psi\rangle_{\text {in }}=\sum_{j=1}^{N} \alpha_{j}|j\rangle_{a}|\emptyset\rangle_{b} \stackrel{\mathrm{qRAM}}{\longrightarrow}|\psi\rangle_{\text {out }}=\sum_{j=1}^{N} \alpha_{j}|j\rangle_{a}\left|D_{j}\right\rangle_{b},
$$

\footnotetext{
*englund@mit.edu
}

Published by the American Physical Society under the terms of the Creative Commons Attribution 4.0 International license. Further distribution of this work must maintain attribution to the author(s) and the published article's title, journal citation, and DOI. where $N$ is the number of memory cells and the subscripts $a$ and $b$ denote the address and bus qubits, respectively. One efficient implementation of qRAM proposed by Giovannetti, Lloyd, and Maccone (GLM) [1,2] is the "bucket-brigade model": a binary tree of memory nodes that direct the bus qubit to the data layer. A tree of depth $n>1$ contains a total of $2^{n}-1$ nodes, with the last layer containing $N=2^{n-1}$ memory cells. Each preceding layer $i$ represents the register $k_{i}$ of the address $|j\rangle=\left|k_{1} k_{2} \cdots k_{n-1} k_{n}\right\rangle$, which sets the path leading to the corresponding memory cell $D_{j}$ [Fig. 1(a)].

Principally, these memory nodes must (1) store an address register qubit that (2) routes ensuing qubits for addressing and retrieval. The register $\left|k_{i}\right\rangle$ sets the internal state of layer $i$, which governs routing of the subsequent registers $\left\{\left|k_{i+1}\right\rangle,\left|k_{i+2}\right\rangle, \ldots\right\}$. A qRAM query thus performs a sequence of alternating state transfer and routing operations, with each register qubit determining how the node routes the subsequent register. Once the binary tree has been programmed by the state of address qubits, $\sum_{j} \alpha_{j}|j\rangle_{a}$, it is traversed by the bus photon $|\downarrow\rangle_{b}$ to access the memory cells $\left\{D_{j}\right\}$ in superposition. The bus qubit travels back up the tree and addresses are mapped 
(a)

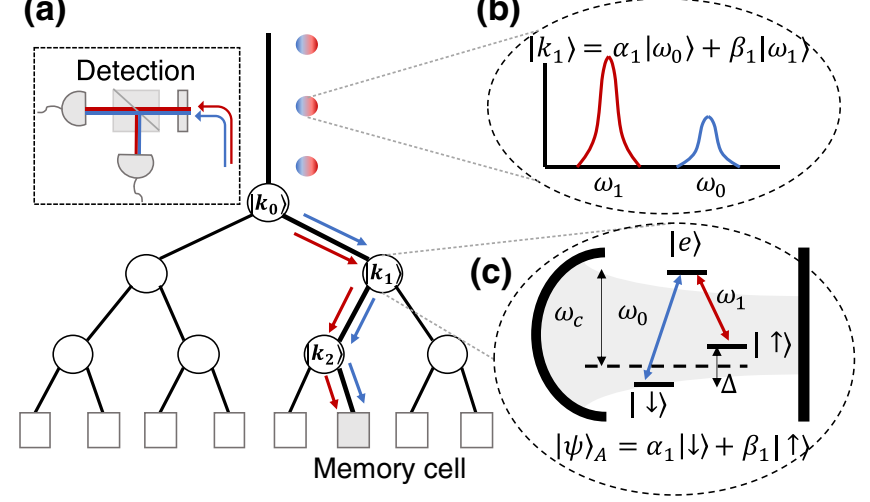

(d)

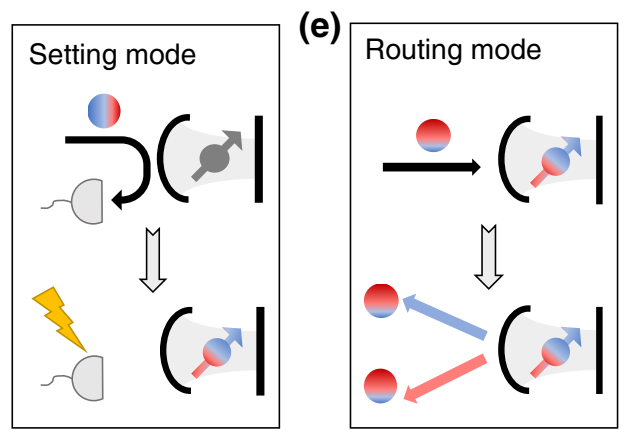

FIG. 1. An illustrative bucket-brigade model with a cavitycoupled $\Lambda$-level atom at each tree node. (a) The address $|j\rangle$ consisting of the register qubits $\left|k_{0}\right\rangle\left|k_{1}\right\rangle\left|k_{2}\right\rangle$ arrives at the threelevel binary tree containing $N=2^{3}$ memory cells. (b) Each register is a frequency-encoded photonic qubit in the $\left\{\omega_{0}, \omega_{1}\right\}$ basis. (c) For our implementation, each tree node is a $\Lambda$-atom coupled to a single-sided nanocavity, the resonant frequency $\omega_{c}$ of which is tuned to the average of the two atomic transition frequencies, $\omega_{0}$ and $\omega_{1}$, which are separated by a Zeeman splitting $\Delta$. For layer 1 , the register $\left|k_{1}\right\rangle$ sets the internal state of the node to $\left|\psi_{A}\right\rangle=\alpha_{1}|\downarrow\rangle+\beta_{1}|\uparrow\rangle$ that routes the successive register $\left|k_{2}\right\rangle$. Two essential operations are (d) the setting mode via cavity reflection and (e) the routing mode.

onto the returning register qubits to disentangle themselves from the nodes, producing the qRAM output state $|\psi\rangle_{\text {out }}$. The ability to perform this operation in $\log (N)$ time steps highlights the advantage of quantum parallelism and offers polynomial speedups in quantum algorithms for applications such as quantum machine learning [3], matrix inversion [4], quantum imaging [5], and quantum searching [6].

Despite its mathematical elegance, no qRAM proposals have been experimentally demonstrated. The existing proposals are based on neutral atoms $[2,7,8]$ and superconducting circuits [9] but still require elementary components to be realized. Here, we introduce a scheme that assembles separately demonstrated technologies into a photonicintegrated-circuit (PIC) architecture integrated with artificial atoms. Namely, the system contains a high-fidelity frequency beam splitter [10-12], nanocavities strongly coupled to long-lived spin memories [13,14], and a scalable nanophotonic Mach-Zehnder interferometer (MZI) array [15]. Importantly, the protocol relies on a cavityassisted controlled-phase $(C Z)$ gate [16] the heralding of which inherently provides the ability to detect qubit loss. We estimate our PIC implementation of the GLM scheme to achieve kilohertz success rate for a qRAM containing $>10^{2}$ memory cells. Furthermore, we propose an alternative approach based on quantum teleportation. This teleportation scheme enables scaling to $10^{5}$ memories while still achieving a greater-than-kilohertz success rate. More importantly, the framework of the protocol applies to quantum networks that require no additional modifications. Thus, our study provides a promising blueprint for building a general qRAM essential for quantum information processing.

\section{BUCKET-BRIGADE SCHEME}

In our PIC implementation, the address register and the bus qubits are the frequency-encoded photons $\left|\psi_{P}\right\rangle=$ $\alpha\left|\omega_{0}\right\rangle+\beta\left|\omega_{1}\right\rangle$ shown in Fig. 1(b) prepared by electrooptic modulators, which together act as a frequency beam splitter [10-12]. They arrive at each node in the binary tree and interact with a cavity-coupled atom, which has two spin states, $|\downarrow\rangle$ and $|\uparrow\rangle$. Both states are coupled to an excited state $|e\rangle$ with respective transition frequencies $\omega_{0}$ and $\omega_{1}$, as shown in Fig. 1(c). In this proposal, we specifically consider the negatively charged diamond siliconvacancy $\left(\mathrm{SiV}^{-}\right)$center, strongly coupled to a single-sided cavity $[13,14]$. By having the cavity resonance $\omega_{c}$ equally detuned from the two transitions, i.e., $\omega_{0,1}=\omega_{c} \pm \Delta / 2$, where $\Delta$ is the Zeeman splitting between the spin states, the resulting Fano interference satisfies the following conditions: upon a cavity reflection, the photon acquires no phase shift when it is resonant with the atomic transition; otherwise, it receives a $\pi$ phase shift (see Appendix A).

This spin-dependent phase shift enables the two operation modes necessitated by the bucket-brigade model: the photonic qubit "setting" the spin state [Fig. 1(d)] and the spin qubit routing the subsequent register qubits [Fig. 1(e)]. The cavity interaction enables a $C Z$ gate for heralding a quantum state transfer between the photonic and the spin qubits, as shown in Fig. 2(a). The very same phase dependence on the atomic state also allows quantum routing by leveraging the cavity system as an interferometer.

Explicitly, in the PIC platform, each node comprises an MZI, an add-drop filter resonant with the $\omega_{0}$ component, and a single-sided nanocavity coupled to an $\mathrm{SiV}^{-}$center. First, in the setting mode, the atom is initialized in a superposition state $\left|\psi_{A}\right\rangle=(|\downarrow\rangle+|\uparrow\rangle) / \sqrt{2}$ by a Hadamard operation. Figure 2(b) shows the register qubit $\left|\psi_{P}\right\rangle=$ $\alpha\left|\omega_{0}\right\rangle+\beta\left|\omega_{1}\right\rangle$ arriving at the MZI and exiting out of the 
(a)

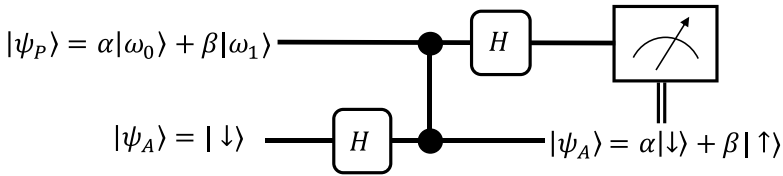

(b)

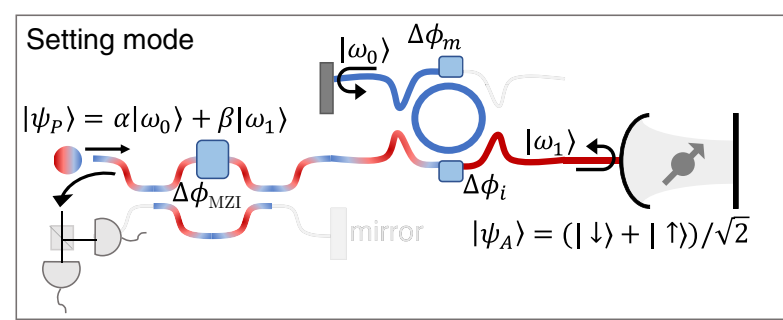

(c)

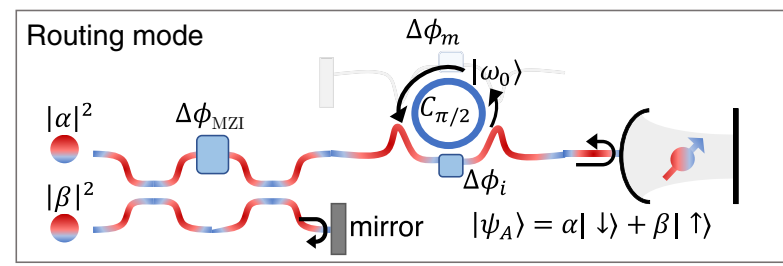

FIG. 2. The PIC implementation of qRAM. (a) The circuit representation of a quantum state transfer operation that maps the register qubit $\left|\psi_{P}\right\rangle$ onto the atomic qubit $\left|\psi_{A}\right\rangle$. (b) In the setting mode, the photon undergoes a $C Z$ operation to complete quantum state transfer. After passing through the MZI, the $\left|\omega_{0}\right\rangle$ component resonantly couples to the add-drop filter that imparts a $\pi$ phase shift upon reflection off the mirror, while the $\left|\omega_{1}\right\rangle$ component interacts with the atom-cavity system and acquires a spin-dependent phase shift. (c) In the routing mode, the MZI is set to a 50:50 beam splitter and the top waveguide of the adddrop filter is decoupled such that the ring resonator imparts a $\pi / 2$ phase shift to the $\left|\omega_{0}\right\rangle$ component upon a single pass. After cavity reflection, the returning photon reinterferes with itself and is routed to either the $|\downarrow\rangle$ path with probability $|\alpha|^{2}$ or the $|\uparrow\rangle$ path with probability $|\beta|^{2}$.

top output port. An add-drop filter then directs the $\omega_{0}$ component to a mirror (e.g., a Sagnac-loop reflector) such that $\left|\omega_{0}\right\rangle$ acquires a $\pi$ phase shift upon reflection regardless of the spin state. On the other hand, the $\omega_{1}$ component continues down the path and reflects off the atom-cavity system, acquiring a spin-dependent phase shift. Finally, the $\omega_{0}$ and $\omega_{1}$ components recombine and undergo a Hadamard transformation by a frequency beam splitter before heralding the completion of quantum state transfer. While heralding conveniently provides the detection of photon loss error, it is essential for the detection system to be shared by all the qRAM layers at the root of the tree. A local detection would otherwise reveal the path information and thereby collapse the superposition of addresses. Therefore, all the register qubits must reflect off the qRAM nodes and return to the root to preserve entanglement between the spin qubits and the address paths. This path erasure also ensures that the same $C Z$ gate applies to all the active nodes in layer $i$ during the setting operation.
After the photon detection, the MZI is switched to a 50:50 beam splitter and the tunable add-drop filter is turned "off" such that the ring resonator only imparts a $\pi / 2$ phase shift to the $\omega_{0}$ component upon a single pass (see Appendix B). Hence, the photon acquires a spin-dependent phase shift independent of the frequency component. As illustrated in Fig. 2(c), the subsequent register qubit $\left|k_{1}\right\rangle$ arrives at the 50:50 beam splitter. One of the MZI output ports connects to the same path as before, while the other leads to a mirror. As a result, the photon taking the former route acquires a spin-dependent phase shift from interacting with the cavity, while one taking the latter route always acquires a $\pi$ phase from reflecting off the mirror. The returning photon then interferes with itself at the beam splitter and is routed to an exit port, depending on the spin state. With $|\alpha|^{2}$ probability, the photon exits out of the top path corresponding to the $|\downarrow\rangle$ spin state; and with $|\beta|^{2}$ probability, it travels down the bottom path corresponding to the $|\uparrow\rangle$ spin state. Effectively, the beam splitter, in conjunction with the atom-cavity system, constitutes an MZI, with the spin-cavity system acting as a phase shifter.

Both the setting and routing operations are repeated alternatingly, carving out the path for the bus qubit to arrive at the desired memory cells. The data can be transferred onto the bus qubit with the same cavity-reflection scheme by reversing the role of the photonic and the spin qubits, followed by a projective measurement on the atom via single-shot readouts [17]. Finally, the sequence is run backward to disentangle the binary tree from the address qubits, leaving the data qubits $\left|D_{j}\right\rangle$ correlated with their respective addresses $|j\rangle$.

\section{A. Setting fidelity per node}

In our cavity-assisted scheme, qubit loss is a heralded error. Therefore, a sequence of successful photon detection guarantees the absence of infidelity stemming from photon loss in the qRAM output. Here, we analyze imperfections in the atom-cavity system that critically affect quantum state transfer as the primary source of infidelity in our protocol, since any inexact mapping from the register qubit to the spin qubit would result in faulty routing of the subsequent registers. To characterize the setting fidelity given an input register $|\psi\rangle_{P}=\alpha\left|\omega_{0}\right\rangle+\beta\left|\omega_{1}\right\rangle$, we calculate the resultant spin state $|\psi\rangle_{A}$ after heralding via a Schrödinger picture evolution:

$$
|\psi\rangle_{A}=\left[2 \alpha r_{m} \pm \beta\left(r_{\mathrm{on}}+r_{\mathrm{off}}\right)\right]|\downarrow\rangle \pm \beta\left(-r_{\mathrm{on}}+r_{\mathrm{off}}\right)|\uparrow\rangle,
$$

where $r_{\text {on }}\left(r_{\text {off }}\right)$ is the on-resonance (off-resonance) cavity reflection and $r_{m}$ is the mirror reflection. Note that $\beta$ is positive (negative) if the photon is detected in the $\omega_{0}\left(\omega_{1}\right)$ port (see Appendix C).

After a $C Z$ gate (see Appendix C), the overlap between the heralded spin state $\left|\psi_{s, f}(i)\right\rangle$ and the target state $|\psi\rangle_{A}=$ 
$\alpha|\downarrow\rangle+\beta|\uparrow\rangle$ defines the state transfer fidelity $\mathcal{F}$, of which we take the average over six representative states $\left|\phi_{i}\right\rangle$ (the axes of a Bloch sphere) [18,19]:

$$
\mathcal{F}=\frac{1}{6} \sum_{i} \mathcal{F}_{i}=\frac{1}{6} \sum_{i}\left|\left\langle\phi_{i} \mid \psi_{s, f}(i)\right\rangle\right|^{2},
$$

where $\quad\left|\phi_{1}\right\rangle=|\downarrow\rangle,\left|\phi_{2}\right\rangle=|\uparrow\rangle,\left|\phi_{3,4}\right\rangle=(|\downarrow\rangle \pm|\uparrow\rangle) / \sqrt{2}$, $\left|\phi_{5,6}\right\rangle=(|\downarrow\rangle \pm i|\uparrow\rangle) / \sqrt{2}$ in the $\{|\downarrow\rangle,|\uparrow\rangle\}$ basis.

Due to the subnanosecond travel times of photonic qubits and conditional gate time on the atoms (approximately $30 \mathrm{~ns}$ based on Ref. [14]) being short relative to the $\mathrm{SiV}^{-}$spin coherence time $T_{2}>10 \mathrm{~ms}$ [17], we will neglect errors caused by spin decoherence. Instead, the performance of the setting mode relies on the coupling strength of the cavity to the output waveguide mode. When the waveguide-cavity coupling is unity, i.e., $\kappa_{\mathrm{wg}} / \kappa=1$, the cavity reflection solely determines the transfer fidelity, which scales as $(C-1) /(C+1)$ in the large-cooperativity limit [20,21]. However, for any reduced $\kappa_{\mathrm{wg}} / \kappa<1$, the need to balance losses becomes especially important. For example, for a desired state $\left|\phi_{3}\right\rangle$ where $\alpha=\beta=1 / \sqrt{2}$, balancing losses entails matching the moduli of the on- and off-resonance cavity reflections $r_{\mathrm{on}} \propto \kappa_{\mathrm{wg}}(C-1) /(C+1)$ and $r_{\mathrm{off}} \propto \kappa_{\mathrm{wg}} / \kappa$ (see Appendix C).

In Fig. 3, we analyze $\mathcal{F}$ as a function of $\kappa_{\mathrm{wg}} / \kappa, C$ and $\delta B$, which is the deviation from the optimal magnetic field $B_{\text {opt }} \propto \sqrt{\gamma \kappa\left[2 C+\kappa\left(\kappa-\kappa_{\mathrm{wg}}\right) / 4-\gamma^{2} / 4\right]}$ for the suitable Fano line shape (see Appendix A). For each point in the fidelity contour, a particular value of $r_{m}$ is chosen to optimize the fidelity, assuming that the mirror is tunable. When $\delta B=-20 \%$, Fig. 3(a) indicates that only a selective range of $C \lesssim 20$ and $\kappa_{\mathrm{wg}} / \kappa \in\{0.83,0.98\}$ result in $\mathcal{F}>0.995$. However, as the magnetic field deviation reduces to $-10 \%$ from the optimum, the transferred state fidelity can exceed 0.999 for a selected range of $C$ and $\kappa_{\mathrm{wg}} / \kappa$. Figure 3(c) shows that at the optimal magnetic field, i.e., $\delta B=0 \%$, the transfer fidelity well exceeds 0.999 for any $C>20$ and $\kappa_{\mathrm{wg}} / \kappa>0.94$. Interestingly, a small region of cooperativities $C<20$ and $\kappa_{\mathrm{wg}} / \kappa<$ 0.94 can still achieve $\mathcal{F}>0.999$ by carefully balancing losses. However, the tolerance to a varying $C$ diminishes as $\kappa_{\mathrm{wg}} / \kappa$ decreases. As $\delta B$ approaches $10 \%$, however, the setting fidelity can no longer reach 0.999 . Its disparity with $\delta B=-10 \%$ stems from the asymmetry exhibited by Fano interference (see Appendix A).

The fidelity considered here is the state transfer fidelity per node, which consequently determines the routing fidelity of the node. If we consider the probability of routing erroneously to be $\epsilon=1-\mathcal{F}$ per node, then the query infidelity $1-\mathcal{F}_{\text {query }}$ scales polylogarithmically with the memory size: $1-\mathcal{F}_{\text {query }} \sim \epsilon \log \left(N_{\text {memories }}\right)^{3}$ [22]. Hence, the qRAM query can be performed with high fidelity as long as $\epsilon \ll 1 / \log \left(N_{\text {memories }}\right)^{3}$. We estimate that our
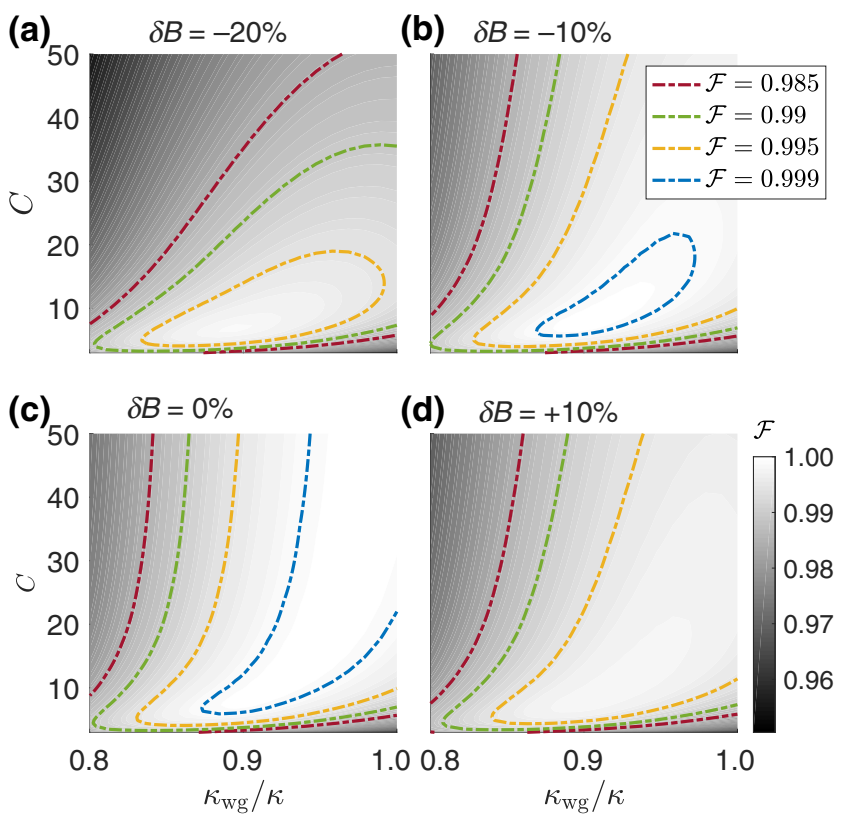

FIG. 3. The quantum state transfer fidelities. The transferred state fidelity for a single setting operation is plotted against the atom-cavity cooperativity $C$ and the waveguide-cavity coupling strength $\kappa_{\mathrm{wg}} / \kappa$ for magnetic field deviations (a) $\delta B=-20 \%$, (b) $-10 \%$, (c) $0 \%$, and (d) $10 \%$. The contour lines denote the fidelity thresholds at $\mathcal{F}=0.985,0.99,0.995$, and 0.999 .

qRAM architecture with state transfer infidelity $\epsilon=10^{-4}$ per node permits querying up to $N_{\text {memories }} \sim 10^{4}$.

\section{B. Efficiency}

Next, we analyze the qRAM query efficiency by first calculating the success probability of heralding each register qubit $\left|k_{i}\right\rangle$ and then the average rate of completing a single query call. Recall that for the bus qubit to reach the memory layer in an $n$-level qRAM, each register photon $\left|k_{i}\right\rangle$ must travel to the node in layer $i \in\{1, \ldots, n\}$ and return to the detector after cavity reflection. Given a propagation loss $\eta_{p}$, the probability of completing the round trip without loss is $e^{-\eta_{p} L(i)}$, where $L(i)$ is twice the distance between the layer $i$ and the root node. However, since the photon can scatter off the single-sided cavity and the mirror into nonwaveguide modes, interaction at each layer further reduces the probability of detecting the returning register qubit by $R_{\mathrm{cav}}$ and $R_{m}$, which represent the cavity and mirror reflection coefficients, respectively. We take their mean reflection coefficient and define the setting efficiency as $\eta_{s}=\eta_{\text {det }}\left(R_{m}+R_{\text {cav }}\right) / 2$, where $\eta_{\text {det }}$ is the detection efficiency. Similarly, the routing efficiency for each layer $i$ would be $\eta_{r}=R_{\text {cav }}$ assuming lossless transmission through the interferometric coupler. As a result, the probability of successfully heralding each register $\left|k_{i}\right\rangle$ 
is

$$
p_{i}=e^{-\eta_{p} L(i)} \eta_{r}^{i-1} \eta_{s} \quad \text { for } i \in\{1, \ldots, n\} .
$$

To calculate the success rate, we must now include both the round-trip travel time to each layer $i$, denoted as $t_{i}=L_{\mathrm{PIC}}(i) / v_{g, \text { PIC }}+L_{\mathrm{dmd}}(i) / v_{g, \mathrm{dmd}}$, where $L_{\mathrm{PIC}}\left(L_{\mathrm{dmd}}\right)$ and $v_{g, \text { PIC }}\left(v_{g, \text { dmd }}\right)$ are the travel distance and group velocity in the PIC (diamond) waveguide. The average time until a successful query call can be found by using the linearity of expectation value. For example, the expected time for a two-level qRAM is

$$
\begin{aligned}
\bar{T}_{n=2}= & p_{1} p_{2}\left(t_{1}+t_{2}\right)+\left(1-p_{1}\right)\left(\bar{T}_{n=2}+t_{1}+\tau_{\text {reset }}\right) \\
& +p_{1}\left(1-p_{2}\right)\left(\bar{T}_{n=2}+t_{1}+t_{2}+\tau_{\text {reset }}\right),
\end{aligned}
$$

where $\tau_{\text {reset }}=5 \mu \mathrm{s}$ is the spin reset time. The first term on the right-hand side is the case of no photons being lost; thus its expected time is simply the product between the success probability of two consecutive heralds $p_{1} p_{2}$ and the total travel time $t_{1}+t_{2}$. The next term represents the case of the $k_{1}$ register photon being lost before detection with probability $1-p_{1}$. Consequently, the average query time $\bar{T}_{n=2}$ is penalized by the additional time $t_{1}+\tau_{\text {reset }}$. Similarly, if the $k_{1}$ photon is heralded but the subsequent register $k_{2}$ is lost with probability $p_{1}\left(1-p_{2}\right), \bar{T}_{n=2}$ is lengthened by $t_{1}+t_{2}+\tau_{\text {reset. }}$. Solving for $\bar{T}_{n=2}$ yields

$$
\bar{T}_{n=2}=\frac{t_{1}+\tau_{\text {reset }}}{p_{1} p_{2}}+\frac{t_{2}}{p_{2}}-\tau_{\text {reset }} .
$$

The expression can be treated as a summation of the round-trip time of each layer weighted by its correspond geometric mean, $-\tau_{\text {reset }}$ since the final trial is a successful run without the need to reset.

We can generalize the average time for a $n$-level qRAM:

$$
\begin{aligned}
\bar{T}= & \left(\prod_{i} p_{i}\right)\left(\sum_{i} t_{i}\right)+\left(1-p_{1}\right)\left(\bar{T}+t_{1}+\tau_{\text {reset }}\right) \\
& +p_{1}\left(1-p_{2}\right)\left(\bar{T}+t_{1}+t_{2}+\tau_{\text {reset }}\right)+\cdots \\
& +\left(\prod_{i}^{n-1}\left(1-p_{n}\right)\right)\left(\bar{T}+\sum_{i} t_{i}+\tau_{\text {reset }}\right) \\
\Rightarrow & \bar{T}=\left(\sum_{i=1}^{n} \frac{t_{i}}{\prod_{j=i}^{n} p_{j}}\right)+\frac{\tau_{\text {reset }}}{\prod_{j=1}^{n} p_{j}}-\tau_{\text {reset }} .
\end{aligned}
$$

Finally, the success rate is then

$$
\bar{\Gamma}=\frac{1}{\bar{T}}
$$

Figure 4(a) shows the qRAM success rate as a function of the number of memories $N_{\text {memories }}=2^{n}$ for different
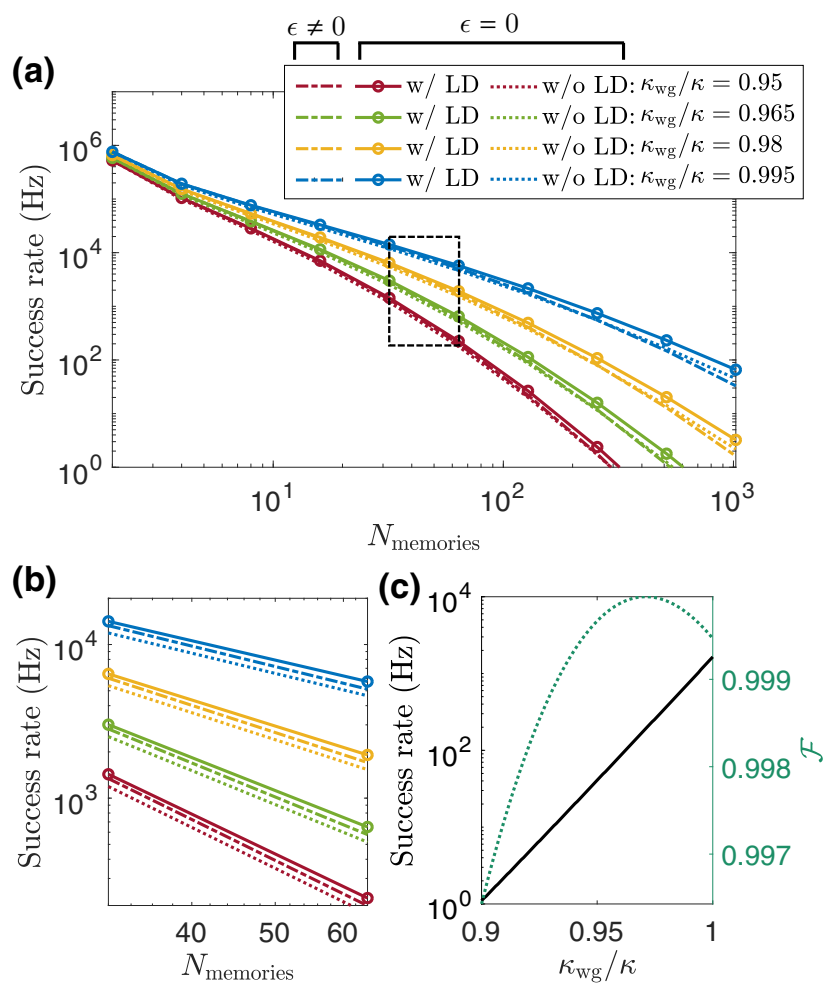

FIG. 4. The efficiency of the PIC qRAM. (a) The success rate (in hertz) is plotted against $N_{\text {memories }}=2^{n}$ for a $n$-level qRAM for $\kappa_{\mathrm{wg}} / \kappa=0.95,0.965,0.98$, and 0.995 for schemes with (solid) and without (dashed) qubit loss detection (LD) with perfect routing operation $(\epsilon=0)$, as well as one with loss detection but with routing error probability $\epsilon=5 \times 10^{-4} \neq 0$ (dashed dotted). On a log-log scale, the success rate rolls off polynomially with increasing $N_{\text {memories }}=2^{n}$ due to an exponentially decreasing success probability of setting each layer $i$ (see Appendix F). (b) An enlargement of the black box in (a), highlighting the slight gain in efficiency for the cavity-assisted scheme with LD. (c) Both the success rate and transfer fidelity vary as a function of $\kappa_{\mathrm{wg}} / \kappa$ for $\epsilon=0$. For a six-level qRAM with $C=100$, there exists a tradeoff between $\bar{\Gamma}$ and $\mathcal{F}$ after $\kappa_{\text {wg }} / \kappa \approx 0.97$ where $\mathcal{F}$ is maximized by perfectly balancing losses.

waveguide-cavity coupling $\kappa_{\mathrm{wg}} / \kappa=0.95,0.965,0.98$, and 0.995 . As $N_{\text {memories }}$ increases, the rates roll off polynomially on the log-log scale, since the success probability $p_{\text {succ }}$ diminishes superexponentially with increasing $n$ (see Appendix F). Furthermore, $p_{\text {succ }}$ intimately depends on the cavity-reflection coefficient $R_{\mathrm{cav}} \propto \kappa_{\mathrm{wg}} / \kappa$, causing $\bar{\Gamma}$ to vary drastically with the waveguide-cavity coupling. For example, the difference between $\kappa_{\mathrm{wg}} / \kappa=0.95$ and $\kappa_{\mathrm{wg}} / \kappa=0.995$ exceeds more than an order of magnitude for $N_{\text {memories }}>10^{2}$ and the disparity grows exponentially as the circuit depth $n$ increases. The unforgiving dropoff in the success rate emphasizes the need for a highly overcoupled single-sided cavity in our protocol.

Additionally, we consider the efficiency for the cases of having perfect $(\epsilon=0)$ and imperfect $\left(\epsilon=5 \times 10^{-4}\right)$ 
routing operation, represented by the solid and the dashed dotted lines, respectively. Recall that if each node has a routing error $\epsilon$, the query error scales as $1-\mathcal{F}_{\text {query }} \sim$ $\epsilon \log \left(N_{\text {memories }}\right)^{3}$ [22]. Hence, the success rate with imperfect routing operations would reduce by an additional factor of $1-\epsilon \log \left(N_{\text {memories }}\right)^{3}$.

On the other hand, the built-in LD of our cavity-assisted scheme enables a slight boost in the success rate. For a scheme without such loss detection, the qRAM must complete the entire sequence of setting and routing all $n$ register qubits before needing to reset, assuming that qubit loss has occurred and been detected after the query. The corresponding success rate would be

$$
\bar{\Gamma}_{\text {no LD }}=\bar{T}_{\text {no LD }}^{-1}=\left(\frac{\sum_{i} t_{i}+\tau_{\text {reset }}}{\prod_{i} p_{i}}-\tau_{\text {reset }}\right)^{-1} .
$$

In contrast, our protocol periodically checks for register losses via photon detection. Therefore, time can be saved by halting and immediately resetting the spins as soon as quantum state transfer fails to herald. Note that the gain in rate, however, depends on the ratio between travel time $t_{i}$ and $\tau_{\text {reset }}$. Figure 4(b) shows a modest increase in success rate for our scheme with $t_{i}<1 \mu \mathrm{s}$ and $\tau_{\text {reset }}=5 \mu \mathrm{s}$ relative to one without loss detection. If $\tau_{\text {reset }} \gg t_{i}$, the slight improvement in efficiency would dwindle as $\bar{\Gamma}$ converges to $\bar{\Gamma}_{\text {no LD. }}$

Lastly, due to the need to balance losses to achieve high transfer fidelity as noted in Sec. II A, there exists an inevitable fidelity-rate trade-off. Given a qRAM containing $2^{6}$ memory cells, Fig. $4(\mathrm{c})$ shows that $\mathcal{F}$ reaches its maximum at $\kappa_{\mathrm{wg}} / \kappa \approx 0.97$ for the assumed cooperativity $C=100$. However, the success rate still increases monotonically with $\kappa_{\mathrm{wg}} / \kappa$ even past this optimum fidelity point. The waveguide-cavity coupling regime in which the trade-off exists narrows with higher atom-cavity cooperativity, since both $\left|r_{\mathrm{on}}\right|$ and $\left|r_{\mathrm{off}}\right|$ increase with $C$ and $\kappa_{\mathrm{wg}} / \kappa$. Nonetheless, at $C=100$ (which has been experimentally demonstrated in Refs. $[13,14])$, the success rate can already exceed $1 \mathrm{kHz}$ while maintaining high fidelity $\mathcal{F}>0.999$.

\section{TELEPORTATION SCHEME}

While the aforementioned scheme is viable for a lowdepth qRAM, the need to sequentially set each address register via cavity reflection inhibits scaling up to $10^{6}$ memories due to photon loss from cavity interaction. Here, we present an alternative approach that writes the address registers onto all the layers simultaneously via quantum teleportation. Crucial to this step is the ability to perform high-fidelity two-qubit gate operation locally between an electron spin (broker qubit) and its neighboring nuclear spin (memory qubit). Considering that gate fidelity $>0.99$ has already been achieved experimentally via composite pulses and optimal classical control [23], we assume unity gate fidelity and success probability for the following calculations and consider the general case in Sec. III B.

The protocol assumes two physically separated entities, a quantum computer (QC) and a qRAM, both containing nodes each of which includes a memory and a broker qubit. The QC contains $n$ nodes that hold the query addresses $\sum_{j} \alpha_{j}|j\rangle_{a}$ stored in the memory qubits. While the memory qubit can only interact with the broker qubit locally, the broker qubit can directly interact with photons to generate spin-photon entanglement via cavity reflection (see Appendix C). Hence, any two nodes can be remotely entangled by having their broker qubits to sequentially interact with a single photon (see Appendix G 1). Subsequently, the two entangled broker qubits undergo a SWAP operation with their memory qubits via hyperfine interaction to preserve coherence (see Appendix G 2). In the same manner, two neighboring Bell states can then be entangled to form a four-qubit Greenberger-Horne-Zeilinger (GHZ) state. Prior to each query operation, all the nodes across each $i$ th $\mathrm{qRAM}$ layer are entangled to generate a $2^{i}$-qubit GHZ state: $\left|\Psi_{i}\right\rangle=(|00 \cdots 0\rangle+|11 \cdots 1\rangle) / \sqrt{2}$. At the same time, the QC and the qRAM are remotely entangled via the same photon-assisted procedure, leaving the QC and the qRAM in the configuration depicted in Fig. 5(a).

Now, each QC node undergoes a local Bell-state measurement (BSM) between its memory and broker qubits as illustrated in Fig. 5(b). As a result, the query addresses are teleported onto all the qRAM layers in parallel. However, since the routing operation depends on the photon interacting with the cavity-coupled broker qubits, each qRAM node undergoes a local SWAP operation immediately before data retrieval [Fig. 5(c)]. The photonic bus qubit then arrives at the binary tree prepared in the state shown in Fig. 5(d).

The data retrieval process remains the same as before. Starting from the root node, the bus photon propagates down the binary tree and is routed based on the statedependent cavity reflection at each layer. After this, the addresses are swapped onto the memory qubits in the qRAM [Fig. 5(e)], followed by remote entanglement between the broker qubits of the QC and the qRAM [Fig. 5(f)]. Then, a local sWAP operation in the QC entangles the memory qubits of the $\mathrm{QC}$ with the broker qubits of the qRAM. Finally, local BSMs in the qRAM teleport the query addresses back onto the QC [Fig. 5(g)], returning the binary tree in its waiting state for future queries [Fig. 5(h)].

Importantly, the proposed architecture extends beyond a PIC platform and can be run on a quantum network, in which each network node represents a tree node in the qRAM. Distillation can be used to generate high-fidelity Bell states [24], which are then joined to form the GHZ states in the same fashion as heralding entanglement links in a quantum repeater. The modularity of the protocol 

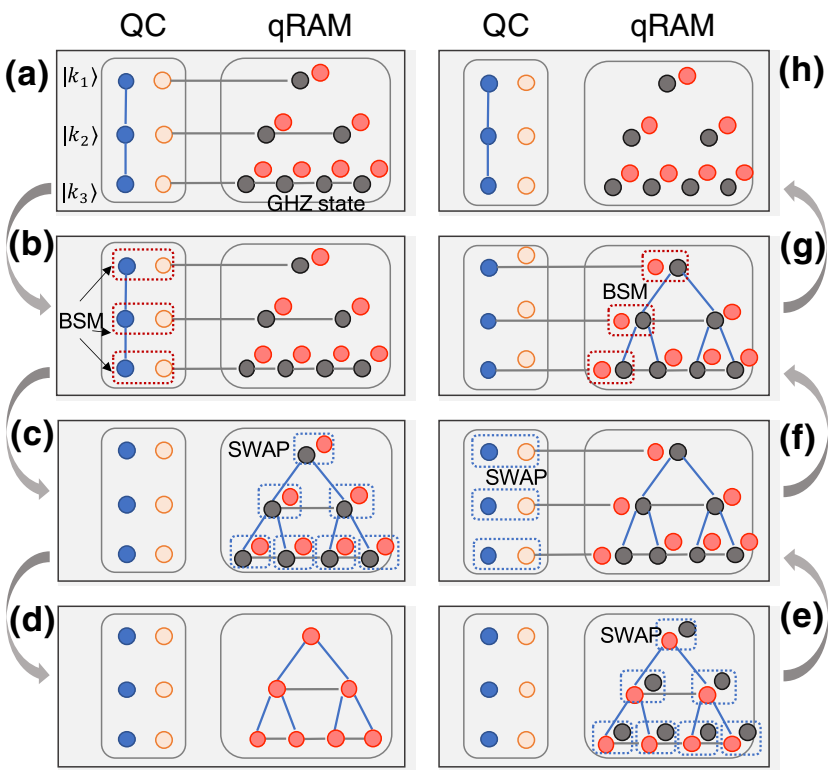

QC memory qubit

QC broker qubit

Data retrieval

O qRAM memory qubit qRAM broker qubit

FIG. 5. The step-by-step procedure of the teleportation scheme. A quantum computer (QC) holds the query addresses that would be mapped onto a qRAM. (a) The QC and qRAM are remotely entangled (as represented by connecting gray lines) and the nodes of each qRAM layer are entangled in a GHZ state. (b) Local Bell-state measurements (BSMs) and subsequent Pauli transformations teleport the query addresses onto the binary tree (c) Then, in each node, the memory (red circle) and the broker (gray circle) qubits undergo a SWAP operation, leaving (d) the qRAM ready for the data retrieval process. (e) After the bus qubit has completed querying, the registers are swapped back onto the memory qubits to maintain coherence. (f) The QC and the qRAM are then remotely entangled again via their broker qubits. A subsequent local SWAP operation in the QC then result in entanglement between the memory qubits of the QC and the broker qubits of the qRAM. (g) Local BSMs in the qRAM then teleport the query addresses back onto the $\mathrm{QC}$, returning $(\mathrm{h})$ the binary tree to its original state.

effectively allows the qRAM query to act as a subroutine for distributed quantum computing.

\section{A. Efficiency comparison}

Here, we compare the efficiency of the two proposed schemes assuming perfect spin-photon gate fidelity $(\epsilon=0)$ via optimally balancing losses. The teleportation approach, similar to the GLM scheme, still requires restarting the query procedure if the bus photon is lost during the retrieval step, since the path information is revealed by the environment. Despite this, the rate of success for the teleportation scheme still scales much more favorably than the GLM approach. Figure 6 compares the query efficiency between the two approaches. For small circuit sizes $<10^{2}$ memories, the GLM scheme achieves higher success rates, since the process of generating GHZ states and remote entanglement links is more costly in time than directly transferring the registers sequentially (see Fig. 14). However, as the qRAM depth increases past the crossover region with approximately $10^{2}-10^{3}$ memories, the efficiency of the GLM scheme rolls off rapidly.

On the other hand, the success rate of the teleportation scheme decreases relatively slowly. Its efficiency is primarily constrained by the retrieval step that succeeds with probability $\propto \eta_{r}^{n}$, as opposed to $\propto \eta_{r}^{n(n-1) / 2} \eta_{s}^{n}$ in the GLM scheme. Its favorable scaling is conducive to increasing the circuit size for general-purpose applications such as quantum machine learning [3]. Our efficiency simulations (see Appendix G 5) show that the teleportation-based approach can theoretically achieve an average $>\mathrm{kHz}$ success rate for a qRAM containing $10^{5}$ memories.

\section{B. Query fidelity}

One drawback of the teleportation approach is decoherence lowering its query fidelity. This is especially problematic for the teleportation scheme because the protocol requires the spin qubits to wait until remote entanglement links are generated between the QC and the qRAM. Specifically, its requirement to prepare a GHZ state, the decoherence rate of which increases linearly with its size, could further worsen the query fidelity. On the other hand, the GLM scheme that sequentially writes the addresses is not constrained by decoherence. As noted previously, the time scales at which the photon traverses through the PIC and the $C Z$ gate is applied are much shorter than the electron spin coherence time. In essence, the teleportation scheme trades fidelity off for a substantial gain in efficiency (see Fig. 6).

Let us consider the worst case where the entirety of the binary tree is active, meaning that all possible addresses are used. Assuming the coherence times of the electron [17] and nuclear spins [25] to be $10^{-2} \mathrm{~s}$ and $10 \mathrm{~s}$, respectively, we estimate the infidelity caused by decoherence to be $<10^{-1}$ for $N_{\text {memories }}=10^{3}$ (see Appendix G 7). Engineering a ${ }^{12} \mathrm{C}$-rich environment [26] could further improve the coherence times and thereby reduce the infidelity.

Other sources of infidelity include depolarization, measurement errors, and imperfect two-qubit interaction between nuclear and electron spins. To simplify the discussion, we combine all types of errors into one collective "physical error rate" $\epsilon$. We propose having interconnects interspersed between the layers that allow for arbitrary routing (see Appendix G6). As a result, for applications that require querying a small subset of possible addresses sparsely spanning the binary tree, only the necessary number of nodes are activated to minimize infidelity caused by physical errors.

However, for applications that require querying most addresses, the physical error rate could quickly decohere the qRAM, since the infidelity rapidly grows as 


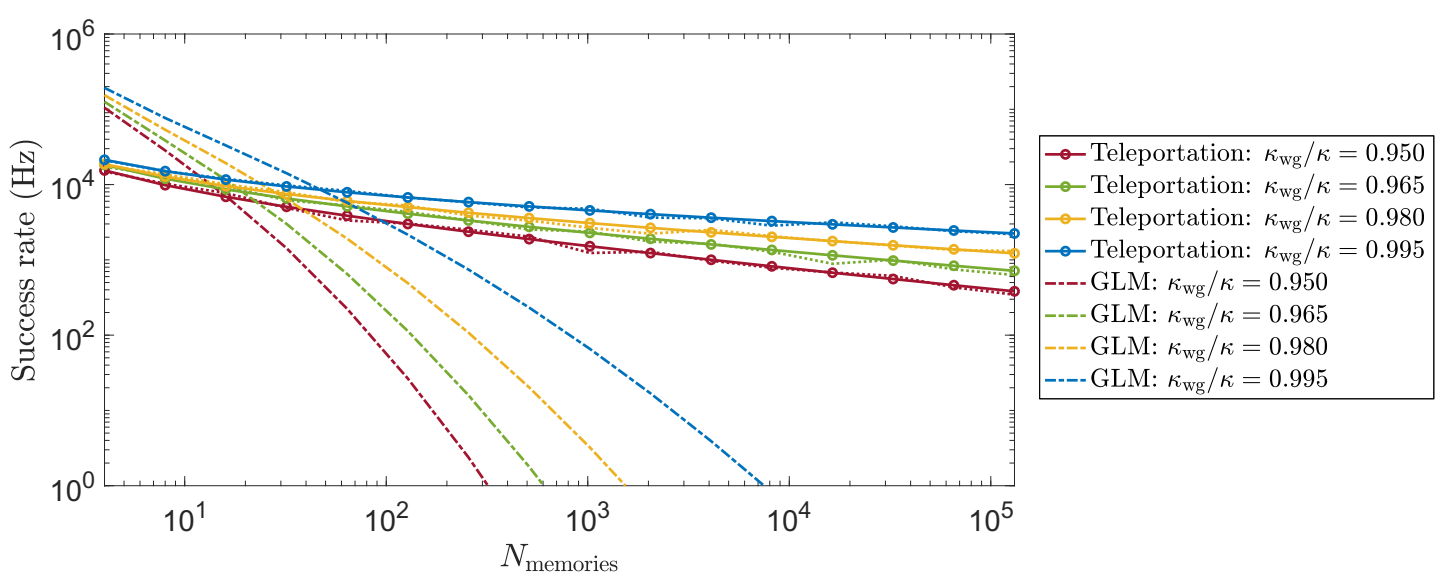

FIG. 6. An efficiency comparison between the conventional GLM scheme (dashed dot) and the teleportation scheme. For the teleportation scheme, the solid lines are analytical fits to the simulation data represented by the dashed lines (see Appendix G 5). Each scheme is evaluated at different cavity-waveguide coupling strengths $\kappa_{\mathrm{wg}} / \kappa=0.95,0.965,0.98$, and 0.995 .

$1-\mathcal{F}_{q} \propto(1-\epsilon)^{2^{n}-1}$ for a circuit depth of $n$. Assuming a physical error rate of $\epsilon=10^{-4}$ and $n=10$, the query infidelity is already approximately $10^{-1}$. Therefore, scaling up of the qRAM necessitates further exploration in converting each tree node to a logical qubit and adapting quantum error correction [27,28].

\section{EXPERIMENTAL CONSIDERATIONS}

Here, we address several experimental considerations concerning inhomogeneity in the solid-state defects.

(1) Spectral diffusion, i.e., slow fluctuation in the optical transitions of the emitter $\left(\omega_{0}, \omega_{1}\right)$, can make it difficult to maintain the cavity resonance at the target $\omega_{c}=\left(\omega_{0}+\omega_{1}\right) / 2$ Fortunately, recent experiments on group IV vacancy centers (including the $\mathrm{SiV}^{-}$center considered here) have shown spectral diffusion far below the radiative line width in nanophotonic waveguides and cavities [14,29]. This stability is due in part to the atomic inversion symmetry of group IV vacancy centers in diamond, which makes optical transitions first-order insensitive to stray electric fields [30]. In particular, recent demonstrations of $\mathrm{SiV}^{-}$centers coupled to cavities have achieved cooperativities $C>100$ [14], which bounds spin-photon gate errors $\propto 1 / C$ (see Appendix A). Further gains in cooperativity are possible with improved cavity designs and reduced environment noise through improved surfaces [31].

(2) Inhomogeneous distribution in the optical transitions $\left(\omega_{0}, \omega_{1}\right)$ (caused by variations in the microscopic environments) would detune the spin-based nodes across the network. Fortunately, these detunings can be corrected by strain tuning, which has been shown to align emitters across nearly the full inhomogeneous distribution $<50 \mathrm{GHz}[29,32]$.

\section{CONCLUSION}

In summary, we introduce a qRAM implementation in a PIC platform integrated with solid-state spin memories. Our numerical simulations show that our architecture can achieve $>0.99$ fidelity with $>\mathrm{kHz}$ success rate for a qRAM containing $10^{2}$ memory cells. Moreover, our cavity-assisted scheme relies on heralding the requisite operations, thereby providing built-in qubit loss detection that further improves the query efficiency. Although high success rates demand a sufficiently overcoupled cavity to the waveguide, existing photonic crystal cavity designs [33-35] already show that they can reach near-unity coupling. We stress that our architecture is technologically feasible given rapidly advancing electro-optic platforms $[36,37]$ and experimentally shown large-scale integration of artificial atoms in PICs [29].

Additionally, we propose an alternative scheme based on quantum teleportation that allows for efficiency scaling favorably with the circuit size. With sufficiently strong cavity-waveguide coupling, the teleportation approach enables a greater-than-kilohertz success rate for a qRAM containing $10^{5}$ memories, a size unattainable by the conventional approach. We emphasize that the protocol is modular and can be applied to a quantum network, in which each network node acts as a tree node in the qRAM. The nodes would again be entangled via heralding, which removes qubit loss as a potential error.

The architecture also extends to other atomic memories: quantum dots [38] and rare-earth ions [39] strongly coupled to nanocavities, and even trapped-ions [40] and neutral atoms [41] suitable for creating large-GHZ states. With rapid advancements in constructing high-fidelity 
atom-photon interfaces, our proposal presents a scalable design of a general qRAM in the NISQ era.

\section{ACKNOWLEDGMENTS}

We thank Dr. Mikkel Heuck and Hyeongrak Choi for insightful discussions. K.C.C. acknowledges funding support by the National Science Foundation Graduate Research Fellowships Program (GRFP), the Army Research Laboratory Center for Distributed Quantum Information (CDQI), and the MITRE Corporation Moonshot program. W.D. is supported by the National Science Foundation to the Computing Research Association for the CIFellows 2020 Program. C.E. acknowledges funding from the Swedish Research Council (Grant No. 201900684). This work was supported by the National Science Foundation QII-TAQS for Quantum Machine Learning with Photonics (Grant No. 1936314).

\section{APPENDIX A: ATOM-CAVITY PARAMETERS}

The reflection of a single-sided cavity coupled with a quantum emitter is

$$
r(\omega)=1-\frac{\kappa_{\mathrm{wg}}\left[i \Delta_{a}+(\gamma / 2)\right]}{\left[i \Delta_{c}+(\kappa / 2)\right]\left[i \Delta_{c}+(\gamma / 2)\right]+g^{2}},
$$

where $g$ is the atom-cavity coupling strength, $\gamma$ is the spontaneous emission rate of the emitter, $\kappa$ is the total decay rate of the cavity, $\kappa_{\mathrm{wg}}$ is the waveguide-cavity coupling rate, and $\Delta_{a}=\omega_{a}-\omega$ and $\Delta_{c}=\omega_{c}-\omega$ are the atomic and cavity detuning from the probe, respectively. In the large-cooperativity $C=4 g^{2} / \kappa \gamma \gg 1$ limit and considering a perfectly overcoupled cavity, the reflection of an on-resonance probe $\Delta_{a}=\Delta_{c}=0$ simplifies to

$$
r(\omega) \stackrel{C \gg 1}{\longrightarrow} \frac{C-1}{C+1} .
$$

Therefore, $r$ approaches +1 when $C$ increases, whereas a far off-resonance emitter decoupled from the cavity mode would yield $r \rightarrow-1$. In our cavity-assisted scheme, the photonic qubits are encoded in the frequency basis $\left\{\omega_{0}, \omega_{1}\right\}$. By appropriately choosing the atomic and cavity detuning, the resultant Fano interference can satisfy the truth table shown in Table I, an entry in which represents the acquired phase of the probe from reflecting off the nanocavity.

This can be satisfied by demanding the reflection to be +1 when the spin state is on resonance and -1 when it is off resonance. Using Eq. (A1), we arrive at the following equation:

$$
\operatorname{Re}\left\{\frac{\kappa_{\mathrm{wg}}\left[i \Delta_{a}+(\gamma / 2)\right]}{\left[i \Delta_{c}+(\kappa / 2)\right]\left[i \Delta_{a}+(\gamma / 2)\right]+g^{2}}\right\}=2 .
$$

TABLE I. The frequency- and spin-dependent phase acquired by the reflected photon.

\begin{tabular}{lcc}
\hline \hline & $|\downarrow\rangle$ & $|\uparrow\rangle$ \\
\hline$\left|\omega_{0}\right\rangle$ & 0 & $\pi$ \\
$\left|\omega_{1}\right\rangle$ & $\pi$ & 0 \\
\hline \hline
\end{tabular}

We center the cavity resonance between the two transition frequencies: $\omega_{c}=\left(\omega_{0}+\omega_{1}\right) / 2$. Therefore, given the Zeeman splitting $\Delta$, the cavity detuning would be half of the spin driving frequency: $\Delta_{c}=\Delta / 2$. Similarly, the atomic detuning would exactly equal the splitting: $\Delta_{a}=$ $\Delta$. In the Purcell regime, Eq. (A3) leads to the following condition:

$$
\Delta \approx \sqrt{2 g^{2}+\frac{\kappa}{4}\left(\kappa-\kappa_{\mathrm{wg}}\right)-\frac{\gamma^{2}}{4}} .
$$

Therefore, given a fixed set of atom-cavity parameters $\left\{g, \gamma, \kappa, \kappa_{\mathrm{wg}}\right\}$, we may set the corresponding magnetic field $B_{\text {opt }}$ that satisfies the appropriate Zeeman splitting $\Delta \sim$ $\mu g B_{\text {opt }} / \hbar$ where $\mu=q \hbar / 2 m_{e}$ is the Bohr magneton and $g \approx 2$ is the Lande $g$ factor.

As an illustrative example, we plot the reflection $r$ of a perfectly overcoupled cavity $\left(\kappa_{\mathrm{wg}} / \kappa=1\right)$ against the probe frequency $\omega / \kappa$. Figure 7(a) shows $r=+1$ at the probe frequency $\omega=\omega_{c}+\Delta / 2$, whereas $r=-1$ at $\omega=$ $\omega_{c}-\Delta / 2$ when the spin population resides in state $|\downarrow\rangle$, and vice versa as shown in Fig. 7(b).

\section{APPENDIX B: FREQUENCY-DEPENDENT ADD-DROP FILTER}

To perform both the (1) setting and (2) routing operations, the add-drop filter must resonantly couple to only the $\omega_{0}$ component to impart (1) a $\pi$ phase shift upon reflection off a mirror and (2) a $\pi / 2$ phase shift through a single pass after decoupling the resonator from the mirror waveguide. The system can be modeled by tracking the evolution of the field propagating through the MZI (or interferometric) couplers [42]. As illustrated in Fig. 8(a), the outputs of the MZI couplers are

$$
\left[\begin{array}{l}
s_{\text {out }} \\
s_{c i-}
\end{array}\right]=\mathcal{T}^{(i)}\left[\begin{array}{c}
s_{\text {in }} \\
s_{c i+}
\end{array}\right], \quad\left[\begin{array}{l}
s_{m+} \\
s_{c m+}
\end{array}\right]=\mathcal{T}^{(m)}\left[\begin{array}{l}
s_{m-} \\
s_{c m-}
\end{array}\right],
$$

where $\mathcal{T}^{(n)}=C^{(n)} Z^{(n)} C^{(n)}$ for $n=\{m, i\}$. The matrices $C^{(n)}$ and $Z^{(n)}$ are transfer matrices that describe the beam splitter and the interferometer arms:

$$
C^{(n)}=\left[\begin{array}{cc}
v_{n} & i \sqrt{1-v_{n}^{2}} \\
i \sqrt{1-v_{n}^{2}} & v_{n}
\end{array}\right], \quad Z^{(n)}=\left[\begin{array}{cc}
e^{i \Psi_{n T}} & 0 \\
0 & e^{i \Psi_{n B}}
\end{array}\right],
$$

where $v_{n}$ represents the coupling to the through waveguide and $\Psi_{n T}$ and $\Psi_{n B}$ are the phases accumulated in 

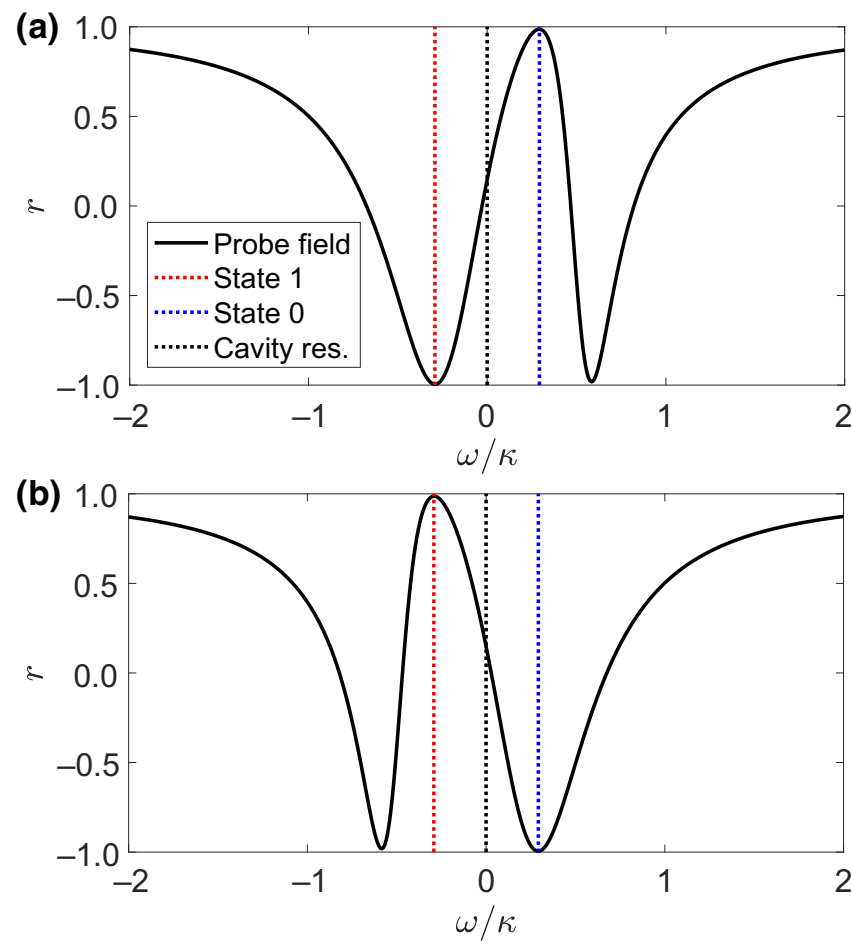

FIG. 7. The cavity reflection as a function of the probe frequency. The normalized probe frequency $\omega / \kappa$ is centered at the cavity resonance (black dashed line) $\omega_{c}$. The magnetic field is appropriately chosen such that the two atomic transition frequencies $\omega_{0}$ and $\omega_{1}$ coincide with the cavity-reflection maximum $r=+1$ and minimum $r=-1$. The reflection when (a) the spin is in the $|\downarrow\rangle$ state is the mirror of when (b) the spin is in the $|\uparrow\rangle$ state.

the phase shifter and the resonator arms, respectively. For the remainder of the section, we assume a balanced interferometric coupler such that $v_{n}=1 / \sqrt{2}$.

Explicitly, we can write the MZI transfer matrix as

$$
\begin{aligned}
\mathcal{T}=e^{i \Psi_{n R}}\left[\begin{array}{cc}
\left(1+e^{i \phi_{n}}\right) v_{n}^{2}-1 & i\left(1+e^{i \phi_{n}}\right) v_{n} \sqrt{1-v_{n}^{2}} \\
i\left(1+e^{i \phi_{n}}\right) v_{n} \sqrt{1-v_{n}^{2}} & v_{n}^{2}-e^{i \phi_{n}}\left(1-v_{n}^{2}\right)
\end{array}\right] \\
\forall n \in\{m, i\},
\end{aligned}
$$

where $\phi_{n}(\omega)=k(\omega) \Delta L_{n}+\Delta \phi_{n}$ and $k(\omega)=\left(n_{\text {eff }} / c\right) \omega_{0}+$ $\left(n_{g, \mathrm{PIC}} / c\right)\left(\omega-\omega_{0}\right)$. Here, $\Delta L_{n}$ is the path-length difference between the two arms and $k(\omega)$ is the propagation constant governed by the effective and group indices in the PIC, $n_{\mathrm{eff}}$ and $n_{g, \text { PIC }}$, respectively.

For the interest of our operations, we can set $s_{\text {in }}=1$ and $s_{m-}=0$. The resultant system of equations is as follows:

$$
\begin{gathered}
s_{\text {out }}=T_{1,1}^{(i)} s_{\text {in }}+T_{1,2}^{(i)} s_{c i+}, \\
s_{c i-}=T_{2,1}^{(i)} s_{\text {in }}+T_{2,2}^{(i)} s_{c i+},
\end{gathered}
$$

(a)
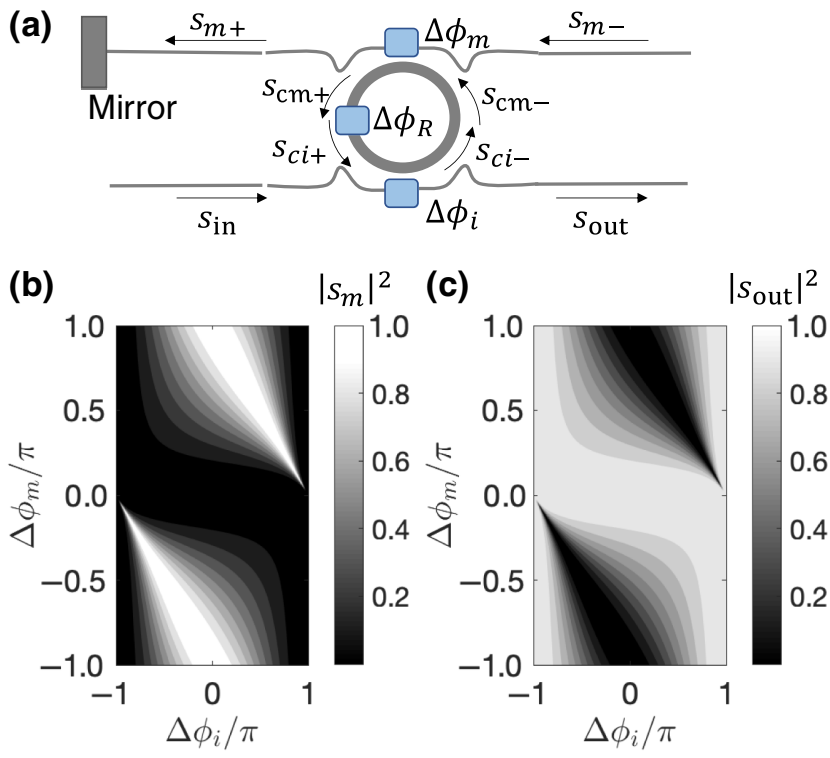

FIG. 8. A schematic of the add-drop filter. (a) Each of the propagating fields in the add-drop filter is labeled for deriving the transfer matrices. The ring resonator (the resonance of which can be tuned by $\Delta \phi_{R}$ ) is coupled to the waveguides via balanced MZI, or interferometric, couplers, each containing a phase shifter $\Delta \phi_{i, m}$. When the top waveguide is coupled to the resonator, the $\omega_{0}$ component is routed to reflect off a Sagnac-loop reflector (mirror). (b) The output intensity toward the mirror $\left|s_{m}\right|^{2}$ as a function of $\Delta \phi_{i}$ and $\Delta \phi_{m}$. (c) The output intensity of the through component $\left|s_{\text {out }}\right|^{2}$.

from which, after solving for $s_{\text {out }}$ and $s_{m+}=T_{1,2}^{(m)} s_{c m-}$, we obtain

$$
\begin{aligned}
s_{\mathrm{out}} & =e^{i \Psi_{i R}}\left(T_{1,1}^{(i)}+\frac{e^{i \phi_{c}} \zeta_{m} T_{1,2}^{\prime(i)} T_{2,1}^{\prime(i)}}{1-e^{i \phi_{c}} \zeta_{i} \zeta_{m}}\right), \\
s_{m+} & =\frac{e^{i \phi_{i m}} T_{1,2}^{(m)} T_{2,1}^{(i)}}{1-e^{i \phi_{c}} \zeta_{i} \zeta_{m}},
\end{aligned}
$$

where $\phi_{c}(\omega)=\psi_{i R}+\phi_{i m}+\psi_{m R}+\phi_{m i}=k(\omega) L_{c}$ is the phase acquired in the resonator and $\zeta_{n}=v_{n}^{2}-e^{i \phi_{n}}(1-$ $\left.v_{n}^{2}\right)$. For the routing operation, we wish to have $s_{m}=$ $s_{m+}=1$ (correspondingly, $s_{\text {out }}=0$ ) such that the $\omega_{0}$ component is entirely directed to the mirror. In Figs. 8(b) and $8(\mathrm{c})$, we plot the output intensity $\left|s_{m}\right|^{2}$ and $\left|s_{\text {out }}\right|^{2}$ as a function of $\Delta \phi_{i}$ and $\Delta \phi_{m}$ set by the phase shifters in the MZI couplers. In order to maximize $\left|s_{m}\right|^{2}$, we find that the phases must satisfy the condition $\Delta \phi_{i}+\Delta \phi_{m}=\pi$.

It is equally essential for the resonator to have a sufficiently high quality factor $(Q)$ such that the line width is narrow enough to only couple to the $\omega_{0}$ instead of both frequencies. For the simulations presented in the main text, the Zeeman splitting is assumed to be approximately $12 \mathrm{GHz}$, which implies that $Q$ must be $>10^{4}$ to resolve between $\omega_{0}$ and $\omega_{1}$. In Fig. 9(a), we find that $\kappa$ is smallest 

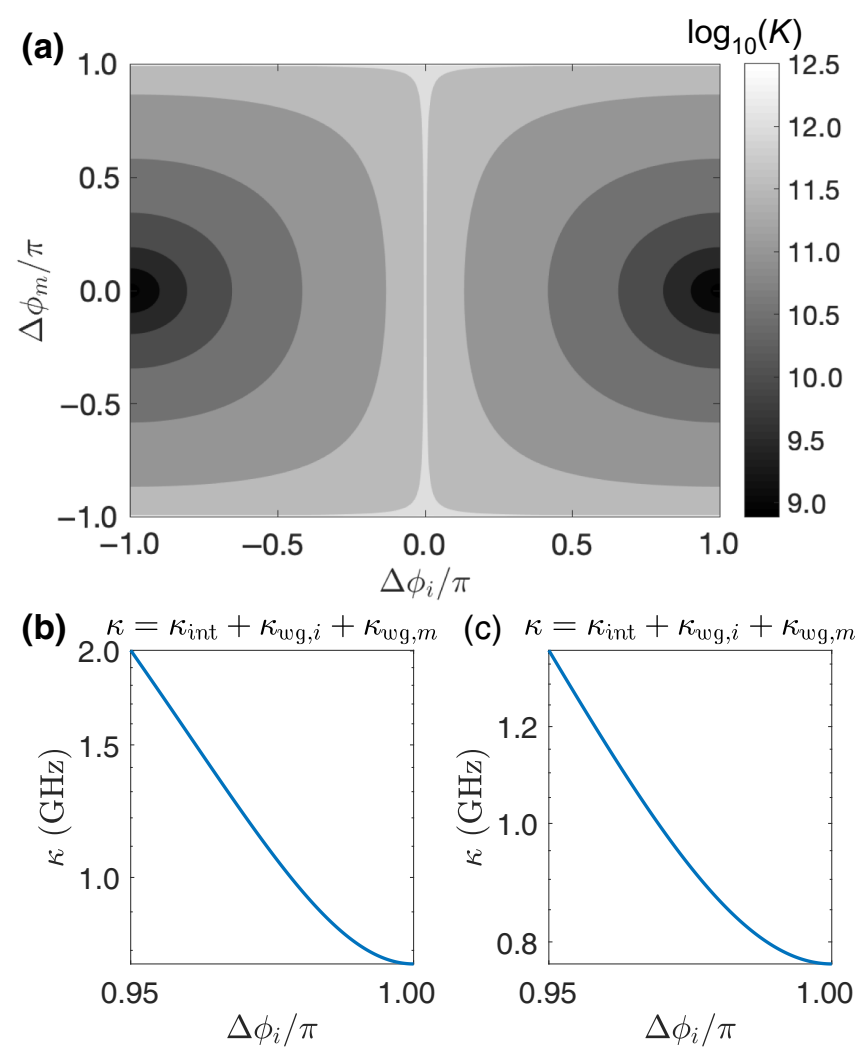

FIG. 9. The decay rate of the ring resonator. (a) The total decay rate (line width) of the resonator is plotted as a function of $\Delta \phi_{i}$ and $\Delta \phi_{m}$ on a log scale. $\kappa$ reaches its minimum near $\Delta \phi_{i}= \pm \pi$ and $\Delta \phi_{m}=0$, at which the resonator is decoupled from the waveguides. $\kappa$ (in gigahertz) is plotted against $\Delta \phi_{i}$ for (b) the setting mode and (c) the routing mode.

at $\Delta \phi_{i}= \pm \pi$, which corresponds to the resonator being completely decoupled from the input waveguide (source) and cavity leakage being maximally suppressed. Similarly, when $\Delta \phi_{m}=0$, the ring (source) is completely decoupled from the mirror waveguide. As long as $\Delta \phi_{i}$ is sufficiently close to $\pi$, Fig. 9(b) indicates that the resonator line width is sufficiently smaller than the Zeeman splitting of approximately $12 \mathrm{GHz}$. For example, at $\Delta \phi_{i}=0.95 \pi$ such that $\Delta \phi_{m}=0.05 \pi,\left|s_{m}\right|^{2}$ is approximately unity and hence satisfies the setting mode. In the routing mode, we only need to minimally shift $\Delta \phi_{m}$ to 0 such that $\left|s_{\text {out }}\right|^{2}=1$ and $\left|s_{m}\right|^{2}=0$, as indicated by the drastically varying region near $\Delta \phi_{i}=\pi$ and $\Delta \phi_{m}=0$, as shown in Figs. 8(b) and $8(\mathrm{c})$. With $\Delta \phi_{m}=0$ fixed, we validate that the narrowness of the resonator line width is as illustrated by Fig. 9(c). $\kappa$ is expectedly smaller in the routing mode than the setting mode, since the resonator is decoupled from the mirror waveguide, thereby having one less leakage channel.

Lastly, we can appropriately choose $\Delta \phi_{R}$, which is the phase shifter within the resonator, such that traversing through the resonator imparts a $\pi / 2$ phase to the $\omega_{0}$ component upon a single pass. In a round-trip, $\left|\omega_{0}\right\rangle$ effectively
TABLE II. After the Pauli X gate on the $\omega_{0}$ component, both frequency modes acquire the same spin-dependent phase.

\begin{tabular}{|c|c|c|c|c|c|c|}
\hline & $|\downarrow\rangle$ & $|\uparrow\rangle$ & & & $|\downarrow\rangle$ & $|\uparrow\rangle$ \\
\hline$\left|\omega_{0}\right\rangle$ & 0 & $\pi$ & $\longrightarrow$ & $\left|\omega_{0}\right\rangle$ & $\pi$ & 0 \\
\hline$\left|\omega_{1}\right\rangle$ & $\pi$ & 0 & $X$ on $\omega_{0}$ & $\left|\omega_{1}\right\rangle$ & $\pi$ & 0 \\
\hline
\end{tabular}

undergoes a Pauli $X$ gate, rendering the truth table shown in Table II.

\section{APPENDIX C: QUANTUM STATE TRANSFER}

\section{Photon-to-spin}

The atom is first initialized in a superposition of the two ground states: $\left|\psi_{A}\right\rangle=(|\downarrow\rangle+|\uparrow\rangle) / \sqrt{2}$. With the incoming frequency-encoded photonic qubit, $\left|\psi_{P}\right\rangle=\alpha\left|\omega_{0}\right\rangle+$ $\beta\left|\omega_{1}\right\rangle$, the joint (unnormalized) photon-atom state is

$$
\begin{aligned}
|\psi\rangle & =\left|\psi_{P}\right\rangle \otimes\left|\psi_{A}\right\rangle \\
& =\left(\alpha\left|\omega_{0}\right\rangle+\beta\left|\omega_{1}\right\rangle\right)(|\downarrow\rangle+|\uparrow\rangle) .
\end{aligned}
$$

The add-drop filter resonantly couples to only the $\omega_{0}$ component, which then reflects off a mirror, acquiring a $\pi$ phase shift regardless of the atomic state. On the other hand, the $\omega_{1}$ component interacts with the atom-cavity system and acquires a spin-dependence phase shift. After the $C Z$ operation, the photon and the atom are entangled:

$$
|\psi\rangle=-\alpha\left|\omega_{0}, \downarrow\right\rangle-\alpha\left|\omega_{0}, \uparrow\right\rangle-\beta\left|\omega_{1}, \downarrow\right\rangle+\beta\left|\omega_{1}, \uparrow\right\rangle .
$$

The returning photon then goes through a frequency beam splitter that performs a Hadamard gate, after which the two frequency components are routed to different photon detectors:

$$
\begin{aligned}
|\psi\rangle= & -\alpha\left(\left|\omega_{0}\right\rangle+\left|\omega_{1}\right\rangle\right)(|\downarrow\rangle+|\uparrow\rangle) \\
& -\beta\left(\left|\omega_{0}\right\rangle-\left|\omega_{1}\right\rangle\right)(|\downarrow\rangle-|\uparrow\rangle) \\
= & \left|\omega_{0}\right\rangle \otimes[-(\alpha+\beta)|\downarrow\rangle-(\alpha-\beta)|\uparrow\rangle] \\
& +\left|\omega_{1}\right\rangle \otimes[-(\alpha-\beta)|\downarrow\rangle-(\alpha+\beta)|\uparrow\rangle] .
\end{aligned}
$$

Upon heralding, the atom undergoes another Hadamard gate to complete quantum teleportation:

$$
\begin{aligned}
|\psi\rangle= & \left|\omega_{0}\right\rangle \otimes[-(\alpha+\beta)(|\downarrow\rangle+|\uparrow\rangle)-(\alpha-\beta)(|\downarrow\rangle-|\uparrow\rangle)] \\
& +\left|\omega_{1}\right\rangle \otimes[-(\alpha-\beta)(|\downarrow\rangle+|\uparrow\rangle) \\
& -(\alpha+\beta)(|\downarrow\rangle-|\uparrow\rangle)] \\
= & -\left|\omega_{0}\right\rangle \otimes(\alpha|\downarrow\rangle+\beta|\uparrow\rangle)+\left|\omega_{1}\right\rangle \otimes(-\alpha|\downarrow\rangle+\beta|\uparrow\rangle) .
\end{aligned}
$$


The end result is

$$
\begin{gathered}
|\psi\rangle=\alpha|\downarrow\rangle+\beta|\uparrow\rangle \quad \text { if } \omega_{0} \text { is detected } \\
\text { or } \quad \alpha|\downarrow\rangle-\beta|\uparrow\rangle \quad \text { if } \omega_{1} \text { is detected, }
\end{gathered}
$$

neglecting global phase. Note that an additional Pauli- $Z$ operation is needed if $\omega_{1}$ is detected.
Now, let us consider an imperfectly overcoupled singlesided cavity with waveguide-cavity coupling $\kappa_{\mathrm{wg}} / \kappa<1$. We denote $r_{\text {off }}$ and $r_{\text {on }}$ as the off- and on-resonance cavity reflections and $r_{m}$ as the mirror reflection. Assuming that the interferometric couplers are lossless in the add-drop filter, the photon-atom entangled state is then

$$
|\psi\rangle=\alpha r_{m}\left|\omega_{0}, \downarrow\right\rangle+\alpha r_{m}\left|\omega_{0}, \uparrow\right\rangle+\beta r_{\text {off }}\left|\omega_{1}, \downarrow\right\rangle+\beta r_{\text {on }}\left|\omega_{1}, \uparrow\right\rangle .
$$

After the Hadamard on the photon,

$$
\begin{aligned}
|\psi\rangle & =\alpha r_{m}\left(\left|\omega_{0}\right\rangle+\left|\omega_{1}\right\rangle\right)(|\downarrow\rangle+|\uparrow\rangle)+\beta\left(\left|\omega_{0}\right\rangle-\left|\omega_{1}\right\rangle\right)\left(r_{\text {off }}|\downarrow\rangle+r_{\text {on }}|\uparrow\rangle\right) \\
& =\left|\omega_{0}\right\rangle \otimes\left[\left(\alpha r_{m}+\beta r_{\text {off }}\right)|\downarrow\rangle+\left(\alpha r_{m}+\beta r_{\text {on }}\right)|\uparrow\rangle\right]+\left|\omega_{1}\right\rangle \otimes\left[\left(\alpha r_{m}-\beta r_{\text {off }}\right)|\downarrow\rangle+\left(\alpha r_{m}-\beta r_{\text {on }}\right)|\uparrow\rangle\right] .
\end{aligned}
$$

The additional Hadamard on the atom would yield

$$
\begin{aligned}
|\psi\rangle= & \left|\omega_{0}\right\rangle \otimes\left[\left(\alpha r_{m}+\beta r_{\text {off }}\right)(|\downarrow\rangle+|\uparrow\rangle)+\left(\alpha r_{m}+\beta r_{\text {on }}\right)(|\downarrow\rangle-|\uparrow\rangle)\right] \\
& +\left|\omega_{1}\right\rangle \otimes\left[\left(\alpha r_{m}-\beta r_{\text {off }}\right)(|\downarrow\rangle+|\uparrow\rangle)+\left(\alpha r_{m}-\beta r_{\text {on }}\right)(|\downarrow\rangle-|\uparrow\rangle)\right] \\
= & \left|\omega_{0}\right\rangle \otimes\left[\left(2 \alpha r_{m}+\beta\left(r_{\text {on }}+r_{\text {off }}\right)\right)|\downarrow\rangle+\beta\left(-r_{\text {on }}+r_{\text {off }}\right)|\uparrow\rangle\right] \\
& +\left|\omega_{1}\right\rangle \otimes\left[\left(2 \alpha r_{m}-\beta\left(r_{\text {on }}+r_{\text {off }}\right)\right)|\downarrow\rangle+\beta\left(r_{\text {on }}-r_{\text {off }}\right)|\uparrow\rangle\right] .
\end{aligned}
$$

If the register qubit is $\left|\psi_{P}\right\rangle=(|\downarrow\rangle+|\uparrow\rangle) / \sqrt{2}$ such that $\alpha=\beta=1 / \sqrt{2}$ and we assume that $\left|r_{m}\right|=1$, detection on the $\omega_{0}$ port would herald the state

$$
|\psi\rangle=\left(2+r_{\text {on }}+r_{\text {off }}\right)|\downarrow\rangle+\left(-r_{\text {on }}+r_{\text {off }}\right)|\uparrow\rangle .
$$

Since $\operatorname{sgn}\left(r_{\text {on }}\right)=1$ and $\operatorname{sgn}\left(r_{\text {off }}\right)=-1$, we see that $|\psi\rangle \Rightarrow$ $|\downarrow\rangle+|\uparrow\rangle$ requires $\left|r_{\text {on }}\right|=\left|r_{\text {off }}\right|$, which hints at the need to "balance" these two reflections. Equation (A1) dictates that $r_{\text {on }} \propto \kappa_{\mathrm{wg}}(C-1) /(C+1)$ while $r_{\text {off }} \propto \kappa_{\mathrm{wg}} / \kappa$, such that only a suitable regime of $\left\{g, \gamma, \kappa, \kappa_{\mathrm{wg}}\right\}$ would maximize the quantum state transfer fidelity as shown in Fig. 3.

\section{Spin-to-photon}

Once the bus qubit retrieves the data from the memory layer, we must extract the address out of the qRAM to obtain the correlated output state $\sum_{j} \alpha_{j}|j\rangle_{a}\left|D_{j}\right\rangle_{b}$. By sending additional photons, we can perform quantum state transfer that maps the spin qubits onto the photonic qubits. Similar to the heralding procedure for transferring the photonic states to spin qubits, the spins must undergo projective measurements to complete the spin-to-photon mapping. While it is feasible to perform single-shot readout on one spin, it is experimentally difficult to simultaneously perform projective measurements on multiple spins within one layer. The issue can be circumvented by introducing an ancillary photon that is entangled with the spins for each layer, and heralding on such photon equates to performing projective readout on the spin qubits.

After data retrieval, the spin holds the routing state $\left|\psi_{A}\right\rangle=\alpha|\downarrow\rangle+\beta|\uparrow\rangle$. The incoming photon initialized in the superposition state (unnormalized) $\left|\psi_{P 1}\right\rangle=\left|\omega_{0}\right\rangle_{1}+$ $\left|\omega_{1}\right\rangle_{1}$ interacts with the cavity, producing the output state

$$
\begin{aligned}
|\Psi\rangle & =-\alpha\left|\omega_{0}\right\rangle_{1}|\downarrow\rangle-\alpha\left|\omega_{1}\right\rangle_{1}|\downarrow\rangle-\beta\left|\omega_{0}\right\rangle_{1}|\downarrow\rangle+\beta\left|\omega_{1}\right\rangle_{1}|\uparrow\rangle \\
& =-\alpha\left(\left|\omega_{0}\right\rangle_{1}+\left|\omega_{1}\right\rangle_{1}\right)|\downarrow\rangle-\beta\left(\left|\omega_{0}\right\rangle_{1}-\left|\omega_{1}\right\rangle_{1}\right)|\uparrow\rangle .
\end{aligned}
$$

After a Hadamard operation on the spin qubit, the entangled state becomes

$$
\begin{aligned}
|\Psi\rangle= & -\alpha\left(\left|\omega_{0}\right\rangle_{1}+\left|\omega_{1}\right\rangle_{1}\right)(|\downarrow\rangle+|\uparrow\rangle) \\
& -\beta\left(\left|\omega_{0}\right\rangle_{1}-\left|\omega_{1}\right\rangle_{1}\right)(|\downarrow\rangle-|\uparrow\rangle) .
\end{aligned}
$$

A subsequent Hadamard operation (via the frequency beam splitter) on the photon yields

$$
\begin{aligned}
|\Psi\rangle= & -\alpha\left|\omega_{0}\right\rangle_{1}(|\downarrow\rangle+|\uparrow\rangle)-\beta\left|\omega_{1}\right\rangle_{1}(|\downarrow\rangle-|\uparrow\rangle) \\
= & -|\downarrow\rangle \otimes\left(\alpha\left|\omega_{0}\right\rangle_{1}+\beta\left|\omega_{1}\right\rangle_{1}\right) \\
& -|\uparrow\rangle \otimes\left(\alpha\left|\omega_{0}\right\rangle_{1}-\beta\left|\omega_{1}\right\rangle_{1}\right) .
\end{aligned}
$$


We then send a subsequent photon $\left|\Psi_{P 2}\right\rangle=\left|\omega_{0}\right\rangle_{2}+\left|\omega_{1}\right\rangle_{2}$ that will entangle with the spin qubit for performing the projective measurement. Similarly, the composite state undergoes a $C Z$ operation upon cavity reflection, resulting in

$$
\begin{aligned}
|\Psi\rangle= & |\downarrow\rangle\left(\left|\omega_{0}\right\rangle_{2}+\left|\omega_{1}\right\rangle_{2}\right)\left(\alpha\left|\omega_{0}\right\rangle_{1}+\beta\left|\omega_{1}\right\rangle_{1}\right) \\
& +|\uparrow\rangle\left(\left|\omega_{0}\right\rangle_{2}-\left|\omega_{1}\right\rangle_{2}\right)\left(\alpha\left|\omega_{0}\right\rangle_{1}-\beta\left|\omega_{1}\right\rangle_{1}\right) .
\end{aligned}
$$

Another Hadamard operation on the second photon would produce an entangled state:

$$
\begin{aligned}
|\Psi\rangle= & |\downarrow\rangle\left|\omega_{0}\right\rangle_{2}\left(\alpha\left|\omega_{0}\right\rangle_{1}+\beta\left|\omega_{1}\right\rangle_{1}\right) \\
& +|\uparrow\rangle\left|\omega_{1}\right\rangle_{2}\left(\alpha\left|\omega_{0}\right\rangle_{1}-\beta\left|\omega_{1}\right\rangle_{1}\right) .
\end{aligned}
$$

As a result, any projection on the frequency-encoded photon is a projective measurement on the spin as well. If $\left|\omega_{0}\right\rangle_{2}$ is detected, the effective projection onto $|\downarrow\rangle$ results in the transferred state onto the first photon. If, instead, $\left|\omega_{1}\right\rangle_{2}$ is detected, an additional $\pi$ pulse would be applied to the first photon to construct $\alpha\left|\omega_{0}\right\rangle_{1}+\beta\left|\omega_{1}\right\rangle_{1}$. Imperfections in the cavity system would be treated in the same fashion as in the previous section by taking account of nonunity reflections: $r_{\text {on }}, r_{\text {off }}$, and $r_{m}$.

\section{APPENDIX D: QUANTUM ROUTING}

In the routing mode, the MZI in Fig. 2(c) is tuned to operate as a 50:50 beam splitter, the unitary matrix of which is denoted by $B$. Let $a$ and $b$ be the annihilation operators for the top and bottom spatial modes such that $a^{\dagger}|0\rangle_{a}|0\rangle_{b}=|1\rangle_{a}|0\rangle_{b}$ represents one photon present in the top waveguide and no photon in the bottom waveguide. The MZI provides the following unitary transformation on the operators:

$$
\begin{aligned}
& B a B^{\dagger}=\frac{1}{\sqrt{2}}(a+i b), \\
& B b B^{\dagger}=\frac{1}{\sqrt{2}}(b+i a) .
\end{aligned}
$$

Assuming input strictly from the top waveguide, our initial state is $\left|\phi_{0}\right\rangle=|1\rangle_{a}|0\rangle_{b}=a^{\dagger}|0\rangle_{a}|0\rangle_{b}$. After passing through the MZI, the state becomes

$$
\begin{aligned}
\left|\phi_{1}\right\rangle & =B\left|\phi_{0}\right\rangle \\
& =B a^{\dagger}|0\rangle_{a}|0\rangle_{b}=B a^{\dagger} B^{\dagger} B|0\rangle_{a}|0\rangle_{b} \\
& =\frac{1}{\sqrt{2}}\left(a^{\dagger}-i b^{\dagger}\right)|0\rangle_{a}|0\rangle_{b} .
\end{aligned}
$$

We can denote the unitary transformation of the atomcoupled cavity system (in conjunction with the resonator) as $P a P^{\dagger}=a e^{i \phi}$. Note that it is only acting on the top waveguide and has no effect on $b$. As a result, the photonic qubit after reflection off the mirror and the cavity system becomes

$$
\begin{aligned}
\left|\phi_{2}\right\rangle & =P\left|\phi_{1}\right\rangle \\
& =\frac{1}{\sqrt{2}}\left(P a^{\dagger}-i b^{\dagger}\right) P^{\dagger} P|0\rangle_{a}|0\rangle_{b} \\
& =\frac{1}{\sqrt{2}}\left(e^{i \phi} a^{\dagger}-i b^{\dagger}\right)|0\rangle_{a}|0\rangle_{b} .
\end{aligned}
$$

Lastly, the photon returns to and interacts with the MZI once again:

$$
\begin{aligned}
\left|\phi_{3}\right\rangle= & B^{\dagger}\left|\phi_{2}\right\rangle \\
= & \frac{1}{\sqrt{2}} B^{\dagger}\left(e^{i \phi} a^{\dagger}-i b^{\dagger}\right) B B^{\dagger}|0\rangle_{a}|0\rangle_{b} \\
= & \frac{1}{2}\left(e^{i \phi}\left(a^{\dagger}+i b^{\dagger}\right)-i\left(b^{\dagger}+i a^{\dagger}\right)\right)|0\rangle_{a}|0\rangle_{b} \\
= & e^{i \phi / 2}\left[\left(\frac{e^{i \phi / 2}+e^{-i \phi / 2}}{2}\right) a^{\dagger}+i\left(\frac{e^{i \phi / 2}-e^{-i \phi / 2}}{2}\right) b^{\dagger}\right] \\
& \times|0\rangle_{a}|0\rangle_{b} \\
= & e^{i \phi / 2}\left[\cos \left(\frac{\phi}{2}\right)|1\rangle_{a}|0\rangle_{b}-\sin \left(\frac{\phi}{2}\right)|0\rangle_{a}|1\rangle_{b}\right] .
\end{aligned}
$$

In summary, the photon exiting the MZI output $a$ [Fig. 2(c)] has a constant $\pi$ phase shift upon reflection off the mirror, whereas one exiting the MZI output $b$ has a spin-dependent $\{0, \pi\}$ phase shift. Together, the MZI-cavity-spin system functions as a spindependent router, mapping $(\alpha|\downarrow\rangle+\beta|\uparrow\rangle) \otimes|0\rangle_{a}|0\rangle_{b}$ to $\alpha|\downarrow\rangle|1\rangle_{a}|0\rangle_{b}+\beta|\uparrow\rangle|0\rangle_{a}|1\rangle_{b}$.

\section{APPENDIX E: PIC IMPLEMENTATION}

Figure 10 illustrates a more detailed schematic of our PIC implementation for each tree node. Specifically, a circulator is appended so that the incoming photon can be routed to the children nodes as opposed to returning to the root.

\section{APPENDIX F: SUCCESS PROBABILITY}

Recall that each register $i$ can be written with success probability $p_{i}$ [Eq. (4)]. A successful qRAM query would consequently occur with a probability that is the product of all the layer probabilities:

$$
p_{\text {succ }}=\prod_{i=1}^{n} p_{i}=e^{-\sum_{i} \eta_{p}(i) L(i)} \eta_{r}^{n(n-1) / 2} \eta_{s}^{n} .
$$

As expected, Fig. 11 shows a polynomial roll-off in the success probability $p_{\text {succ }}$ as the number of memory cells 

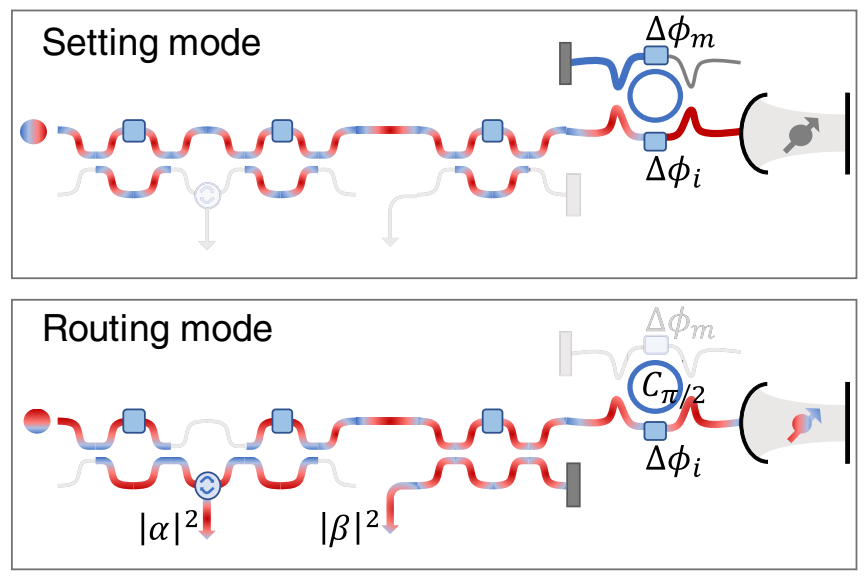

FIG. 10. A detailed PIC implementation of a tree node. Paths that are inactive in each mode are faded out.

$N_{\text {memories }}=2^{n}$ increases. Since the waveguide-cavity coupling $\kappa_{\mathrm{wg}} / \kappa$ mainly determines the cavity reflection, $p_{\text {succ }}$ can differ by orders of magnitude and the difference increases with $N_{\text {memories }}$.

\section{APPENDIX G: TELEPORTATION SCHEME}

Essential to the setup of the teleportation scheme is to create a GHZ state for each layer prior to quantum teleportation. Below, we break down its creation process into three critical steps: photon-assisted Bell-state creation, Bell-state swap between nuclear (memory) and electron (broker) spins, and GHZ-state creation by joining adjacent pairs. After this, we explain how a BSM can be made on two remotely entangled spins via the photonassisted cavity interaction. Lastly, we provide an example of how teleportation enables transferring addresses onto the qRAM.

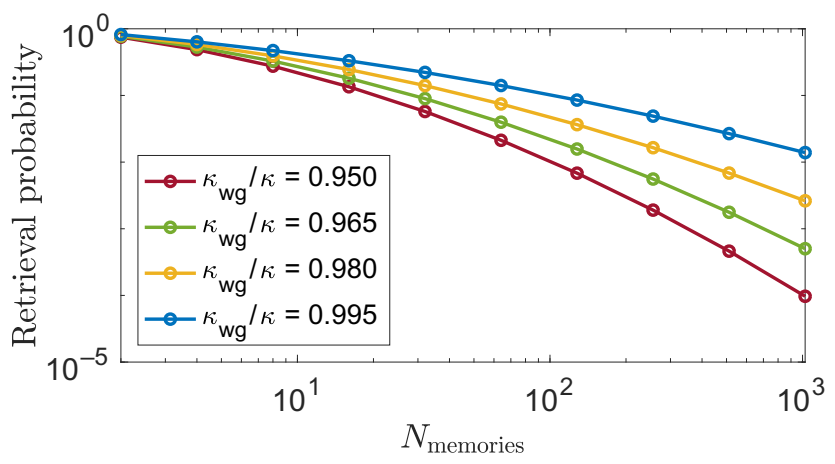

FIG. 11. The retrieval probability as a function of the number of memories on a log-log scale. $p_{\text {succ }}$ displays a polynomial rolloff as $N_{\text {memories }}$ increases for waveguide-cavity coupling $\kappa_{\mathrm{wg}} / \kappa=$ $0.95,0.965,0.98$, and 0.995 .

\section{Photon-assisted Bell-state creation}

In order to create a Bell state between neighboring matter qubits, a photon is sent to reflect off each cavity consecutively. Importantly, the node is in the "setting" mode such that reflection off the cavity system generates a $C Z$ gate. Here, we provide an example of how a photon interacting with two cavities aids construction of a Bell state between the two spin qubits. We begin with the photonic and the spin qubits prepared in the $|+\rangle$ state such that composite state is

$$
|\psi\rangle=\left(\left|\omega_{0}\right\rangle+\left|\omega_{1}\right\rangle\right)\left(|\downarrow\rangle_{1}+|\uparrow\rangle_{1}\right)\left(|\downarrow\rangle_{2}+|\uparrow\rangle_{2}\right),
$$

where the subscripts 1 and 2 denote different spins.

After the photon reflects off the first spin qubit coupled to the cavity, the state becomes an entangled state:

$$
\begin{aligned}
|\psi\rangle= & -\left[\left(\left|\omega_{0}\right\rangle+\left|\omega_{1}\right\rangle\right)|\downarrow\rangle_{1}\right. \\
& \left.+\left(\left|\omega_{0}\right\rangle-\left|\omega_{1}\right\rangle\right)|\uparrow\rangle_{1}\right]\left(|\downarrow\rangle_{2}+|\uparrow\rangle_{2}\right) \\
= & -\left[\left(\left|\omega_{0}\right\rangle|\downarrow\rangle_{2}+\left|\omega_{1}\right\rangle|\downarrow\rangle_{2}+\left|\omega_{0}\right\rangle|\uparrow\rangle_{2}+\left|\omega_{1}\right\rangle|\uparrow\rangle_{2}\right)|\downarrow\rangle_{1}\right. \\
& +\left(\left|\omega_{0}\right\rangle|\downarrow\rangle_{2}-\left|\omega_{1}\right\rangle|\downarrow\rangle_{2}+\left|\omega_{0}\right\rangle|\uparrow\rangle_{2}\right. \\
& \left.\left.-\left|\omega_{1}\right\rangle|\uparrow\rangle_{2}\right)|\uparrow\rangle_{1}\right] .
\end{aligned}
$$

Upon reflecting off the second cavity system, it produces the state

$$
\begin{aligned}
|\psi\rangle= & \left\{\left[\left(\left|\omega_{0}\right\rangle+\left|\omega_{1}\right\rangle\right)|\downarrow\rangle_{2}+\left(\left|\omega_{0}\right\rangle-\left|\omega_{1}\right\rangle\right)|\uparrow\rangle_{2}\right]|\downarrow\rangle_{1}\right. \\
& \left.++\left[\left(\left|\omega_{0}\right\rangle-\left|\omega_{1}\right\rangle\right)|\downarrow\rangle_{2}\left(\left|\omega_{0}\right\rangle+\left|\omega_{1}\right\rangle\right)|\uparrow\rangle_{2}\right]|\uparrow\rangle_{1}\right\} .
\end{aligned}
$$

A Hadamard operation on the photon leads to the final state:

$$
|\psi\rangle=\left|\omega_{0}\right\rangle(|\downarrow \downarrow\rangle+|\uparrow \uparrow\rangle)+\left|\omega_{1}\right\rangle(|\downarrow \uparrow\rangle+|\uparrow \downarrow\rangle) .
$$

If the $\omega_{0}$ detection port clicks, the Bell state $\left|\Phi^{+}\right\rangle=$ $|\downarrow \downarrow\rangle+|\uparrow \uparrow\rangle$ is heralded. On the other hand, if the $\omega_{1}$ port registers a click, the Bell state $\left|\Psi^{+}\right\rangle=|\downarrow \uparrow\rangle+|\uparrow \downarrow\rangle$ is created. A Pauli $X$ gate can be applied to the second spin qubit to transform $\left|\Psi^{+}\right\rangle$to $\left|\Phi^{+}\right\rangle$.

Multiple pairs of adjacent tree nodes can simultaneously undergo the aforementioned evolution to create Bell states. Then, the entangled spin-qubit pairs can be linked by the same procedure. As opposed to having a single photon reflecting off all the nodes across each layer to create a GHZ-like state, a process that inevitably suffers from exponentially decaying success probability, the pairwise creation protocol described here is much more efficient.

\section{Bell-state swap between electron and nuclear spins}

Figure 12(a) shows two electron spins $e L$ and $e R$ entangled in a Bell state: $|00\rangle_{e}+|11\rangle_{e}$. Let the nuclear spins 
(a)

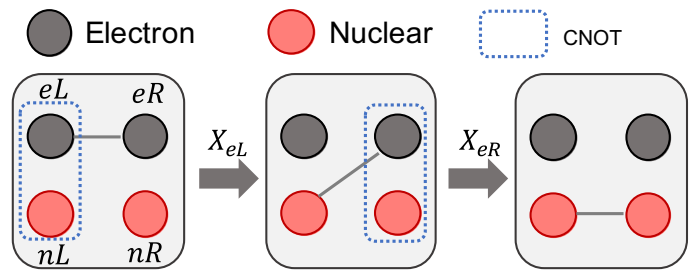

(b)

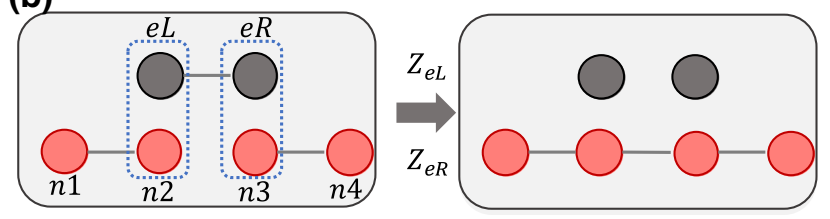

FIG. 12. Operations to: (a) swap a Bell state between a pair of entangled electron spins and a pair of nuclear spins; (b) entangle two pairs of Bell states to form a four-qubit GHZ state in the nuclear spins.

initialized in the ground state $|0\rangle_{n L / n R}$. A controlled NOT (CNOT) operation, where $e L$ acts as the control and $n L$ as the target, yields an effective GHZ state: $|0\rangle_{n L}|00\rangle_{e}+$ $|1\rangle_{n L}|11\rangle_{e}$. Then, an $X$ measurement on $e L$ disentangles the electron spin from the GHZ state, leaving the final state $|\psi\rangle$ :

$$
\begin{aligned}
|\psi\rangle & =\left(\left\langle0| \pm\langle 1|)_{e L}\left(|0\rangle_{n L}|00\rangle_{e}+|1\rangle_{n L}|11\rangle_{e}\right)\right.\right. \\
& =|0\rangle_{n L}|0\rangle_{e R} \pm|1\rangle_{n L}|1\rangle_{e R} .
\end{aligned}
$$

Similarly, a CNOT operation between $e R$ and $n R$ produces $|0\rangle_{n L}|0\rangle_{e R}|0\rangle_{n R} \pm|1\rangle_{n L}|1\rangle_{e R}|1\rangle_{n R}$. A subsequent $X$ measurement on $e R$ then leaves a Bell state between the nuclear spins:

$$
\begin{aligned}
|\psi\rangle & =\left(\left\langle0| \pm\langle 1|)_{e R}\left(|0\rangle_{e R}|00\rangle_{n} \pm|1\rangle_{e R}|11\rangle_{n}\right)\right.\right. \\
& =|00\rangle_{n} \pm|11\rangle_{n} .
\end{aligned}
$$

\section{GHZ-state creation}

Now, we assume that two adjacent pairs of nuclear spins, $\{n 1, n 2\}$ and $\{n 3, n 4\}$, are entangled in a Bell state, as shown in Fig. 12(b). The corresponding electron spins of $n 2$ and $n 3$ are also entangled in a Bell state via a photon-assisted interaction. We first consider the composite state including $n 1, n 2, e L$, and $e R$ after a CNOT operation between $n 2$ and $e L$, in which $n 2$ is the control and $e L$ is the target:

$$
\begin{aligned}
|\psi\rangle= & |00\rangle_{n}|00\rangle_{e}+|00\rangle_{n}|11\rangle_{e}+|11\rangle_{n}|10\rangle_{e}+|11\rangle_{n}|01\rangle_{e} \\
= & \left(|00\rangle_{n}|0\rangle_{e R}+|11\rangle_{n}|1\rangle_{e R}\right)|0\rangle_{e L} \\
& +\left(|00\rangle_{n}|1\rangle_{e R}+|11\rangle_{n}|0\rangle_{e R}\right)|1\rangle_{e L},
\end{aligned}
$$
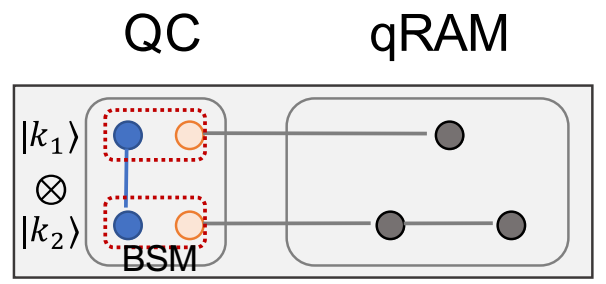

FIG. 13. A two-level qRAM is first entangled with a remote QC. Local BSMs in the QC complete quantum teleportation of the query addresses onto the binary tree. The memory layer is not shown in the schematic for simplicity.

where $|i j\rangle_{e}=|i\rangle_{e L}|j\rangle_{e R}$. A subsequent $Z$ measurement on $e L$ followed by a conditional Pauli transformation on $e R$ yields a GHZ state: $|00\rangle_{n}|0\rangle_{e R}+|11\rangle|1\rangle_{e R}$.

Then, similarly, a CNOT operation between $n 3$ and $e R$ followed by a $Z$ measurement on $e R$ yields the final GHZ state (conditional Pauli transformation on the nuclear spins):

$$
|\psi\rangle=|0000\rangle_{n}+|1111\rangle_{n}
$$

\section{Teleportation}

We present here an example of mapping two-register addresses $\sum_{j} \alpha_{j}\left|k_{1, j} k_{2, j}\right\rangle$ onto a two-level binary tree. Suppose that the query addresses compose the superposition state, $\alpha|00\rangle+\beta|01\rangle+\gamma|10\rangle+\delta|11\rangle$, where each register represents the state of the corresponding node at each tree level. We consider the formalism that the atomic state $|0\rangle$ routes the subsequent qubit to the left branch and $|1\rangle$ to the right. For an instance, the address $|01\rangle$ means that the root (level-1) node is in the state $|0\rangle$ and that the left node of level 2 is in the state of $|1\rangle$.

Each layer in the qRAM is initialized as a GHZ state, e.g., $(|\tilde{0}\rangle+|\tilde{1}\rangle) / \sqrt{2}$, where $|\tilde{i}\rangle=|i i \cdots i\rangle$. Importantly, the first register of each GHZ state belongs to an ancillary qubit in the QC, as shown in Fig. 13.

The unnormalized composite state would then be

$$
\begin{array}{r}
|\Psi\rangle=(\alpha|00\rangle+\beta|01\rangle+\gamma|10\rangle+\delta|11\rangle) \\
\otimes(|00\rangle+|11\rangle)_{1}(|000\rangle+|111\rangle)_{2},
\end{array}
$$

where the subscripts 1 and 2 denote the layer number.

The state can be rewritten as

$$
\begin{aligned}
|\Psi\rangle= & \alpha\left[\left(\left|\Phi^{+}\right\rangle+\left|\Phi^{-}\right\rangle\right)|0\rangle+\left(\left|\Psi^{+}\right\rangle+\left|\Psi^{-}\right\rangle\right)|1\rangle\right]_{1} \\
& \otimes\left[\left(\left|\Phi^{+}\right\rangle+\left|\Phi^{-}\right\rangle\right)|00\rangle+\left(\left|\Psi^{+}\right\rangle+\left|\Psi^{-}\right\rangle\right)|11\rangle\right]_{2} \\
& +\beta\left[\left(\left|\Phi^{+}\right\rangle+\left|\Phi^{-}\right\rangle\right)|0\rangle+\left(\left|\Psi^{+}\right\rangle+\left|\Psi^{-}\right\rangle\right)|1\rangle\right]_{1} \\
& \otimes\left[\left(\left|\Psi^{+}\right\rangle-\left|\Psi^{-}\right\rangle\right)|00\rangle+\left(\left|\Phi^{+}\right\rangle-\left|\Phi^{-}\right\rangle\right)|11\rangle\right]_{2}
\end{aligned}
$$




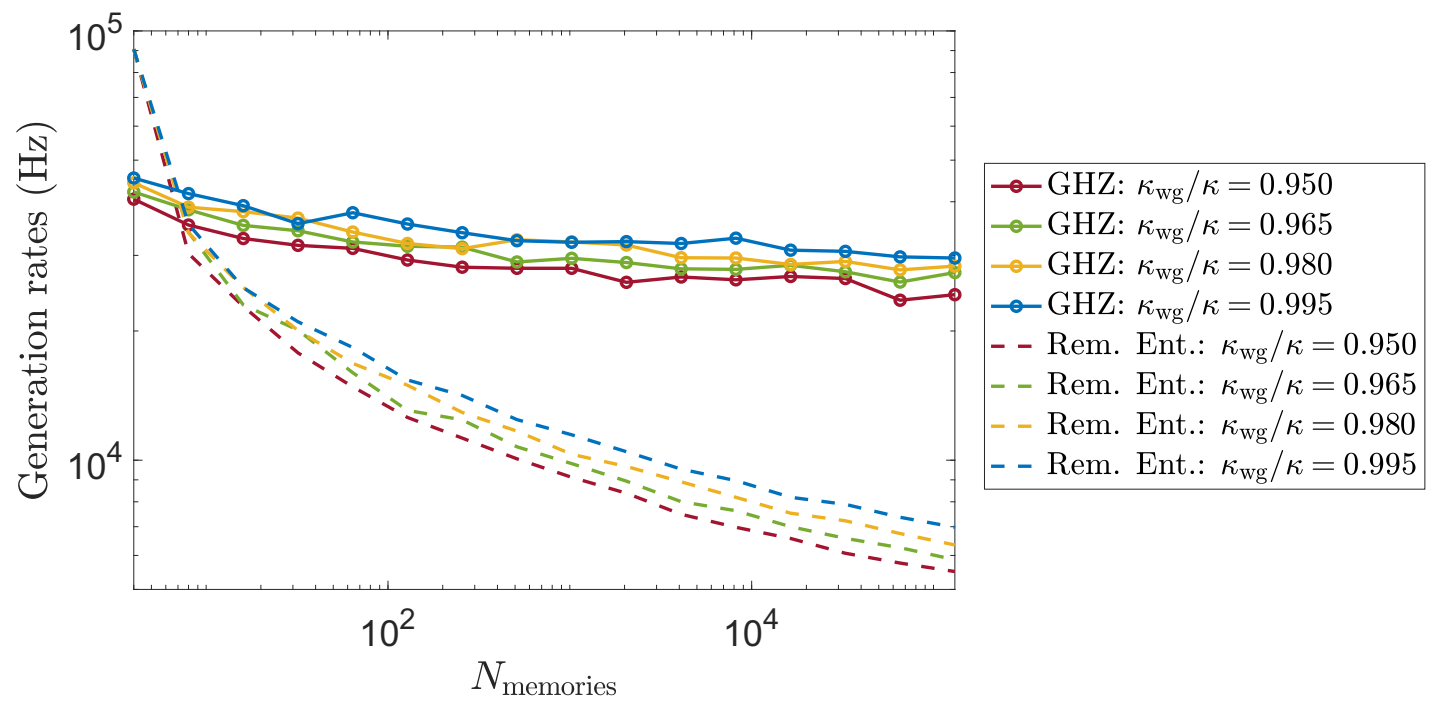

FIG. 14. The generation rates for the GHZ state and the remote entanglement link are evaluated at different cavity-waveguide coupling strengths $\kappa_{\mathrm{wg}} / \kappa=0.95,0.965,0.98$, and 0.995 .

$$
\begin{aligned}
& +\gamma\left[\left(\left|\Psi^{+}\right\rangle-\left|\Psi^{-}\right\rangle\right)|0\rangle+\left(\left|\Phi^{+}\right\rangle-\left|\Phi^{-}\right\rangle\right)|1\rangle\right]_{1} \\
& \otimes\left[\left(\left|\Phi^{+}\right\rangle+\left|\Phi^{-}\right\rangle\right)|00\rangle+\left(\left|\Psi^{+}\right\rangle+\left|\Psi^{-}\right\rangle\right)|11\rangle\right]_{2} \\
& +\delta\left[\left(\left|\Psi^{+}\right\rangle-\left|\Psi^{-}\right\rangle\right)|0\rangle+\left(\left|\Phi^{+}\right\rangle-\left|\Phi^{-}\right\rangle\right)|1\rangle\right]_{1} \\
& \otimes\left[\left(\left|\Psi^{+}\right\rangle-\left|\Psi^{-}\right\rangle\right)|00\rangle+\left(\left|\Phi^{+}\right\rangle-\left|\Phi^{-}\right\rangle\right)|11\rangle\right]_{2} \\
& =\left|\Phi^{+}\right\rangle_{1}\left|\Phi^{+}\right\rangle_{2}\left(\alpha|0\rangle_{1}|00\rangle_{2}+\beta|0\rangle_{1}|11\rangle_{2}\right. \\
& \left.+\gamma|1\rangle_{1}|00\rangle_{2}+\delta|1\rangle_{1}|11\rangle_{2}\right)+\cdots
\end{aligned}
$$

BSMs for each layer would then project the composite state into one of the 16 possible combinations. Followed by conditional Pauli transformations, the query addresses are finally teleported onto the binary tree.

\section{Efficiency simulations}

The teleportation scheme includes four steps: (1) initializing the entanglement links, (2) teleporting the addresses to the qRAM, (3) querying, and (4) teleporting the addresses back to the QC. We perform event-based simulations to estimate the time of completing all four steps.

In step (1), all the nodes except the leftmost node within each qRAM layer are entangled to form a GHZ state. It is created by heralded entanglement between nearestneighbor pairs with success probability $p_{\text {ep }}=\eta_{\text {path }} \eta_{s}^{2} \eta_{\text {det }}$ (ep refers to entangled pair) (see Sec. F). If the entanglement attempt fails, the spins undergo reinitialization for $\tau_{\text {reset }}=5 \mu \mathrm{s}$. If it succeeds, the electron spins (broker qubits) are swapped with their respective nuclear spins (memory qubits), an operation that we assume to take $t_{e \rightarrow n}=16 \mu \mathrm{s}$. Then, the unlinked neighbors are subsequently entangled in the same fashion. To reduce computational costs, we assume that the rate is limited by the largest layer and only simulate its GHZ-state creation process.

Simultaneously, in step (2), we attempt to generate entanglement between the broker qubit of the QC and the leftmost node of the qRAM for each layer. Once the entanglement link is generated, the electron and nuclear spins are again swapped. In simulation, we take the maximum between the time to generate a GHZ state and the time to produce QC-qRAM Bell state. The generation rates for both the GHZ state and the remote entanglement link are plotted in Fig. 14. Once both states are constructed, the leftmost node is entangled with the GHZ state composed of the remaining nodes within the same layer. Then, a local BSM is made between the address register and the QC ancillary qubits. To fairly compare the efficiency of the teleportation scheme with that of the GLM scheme, we neglect the physical distance between the QC and the qRAM in Fig. 6.

In step (3), a bus photon arrives at the root node of the binary tree and is routed to the memory layer with the query success probability $p_{i}$ for an $i$-level qRAM. Finally, in step (4), a QC-qRAM Bell state is constructed again for each layer with probability $p_{\text {ep }}$, followed by local BSMs on the leftmost nodes in the qRAM.

In Fig. 6, the simulation data are plotted along with their analytical fits. Recall that the GHZ states are produced by linking multiples of Bell pairs. If each Bell-pair creation succeeds with probability $p$, it would take a geometric mean of $1 / p$ attempts. In the case of $p=1$, the GHZ-state creation process would merely be a two-step process. For example, for a layer with four nodes, nodes 1 and 2 as well 
TABLE III. The parameter values.

\begin{tabular}{lc}
\hline \hline$\omega_{c}$ & $406.774 \mathrm{THz}$ \\
$\kappa$ & $20.34 \mathrm{GHz}[14]$ \\
$\gamma$ & $94 \mathrm{MHz}$ \\
$\tau_{\text {reset }}$ & $5 \mu \mathrm{s}$ \\
$\eta_{\text {str }}$ & $2.7 \mathrm{~dB}[37]$ \\
$\eta_{\text {bend }}$ & $9.3 \mathrm{~dB}[37]$ \\
$\eta_{\text {det }}$ & $1.3 \mathrm{~dB}[11]$ \\
$R_{\text {resonator }}$ & $50 \mu \mathrm{m}[36]$ \\
$n_{\text {eff }}$ & 2.2645 \\
$n_{g, \text { PIC }}$ & 2.3862 \\
$n_{g, \text { dmd }}$ & 2.4513 \\
$t_{t \rightarrow n}$ & $16 \mu \mathrm{s}$ \\
$t_{n \rightarrow e}$ & $30 \mathrm{~ns}$ \\
$a$ & 1.7094 \\
$b$ & 0.79386 \\
\hline \hline
\end{tabular}

as nodes 3 and 4 are entangled in the first time step. Then, nodes 2 and 3 are entangled to complete the GHZ state creation. However, with a nonunity $p$, the GHZ-state creation is ultimately limited by the pair that fails the most number of times. In other words, the rate is mainly determined by the outlier. We fit the guessed model $f(N)=a N^{-b}$ multiplied with the analytical rate (based on geometric mean) to the simulation data, where $N$ is the number of nodes within the largest layer. The coefficients $a$ and $b$ capture the scaling of the outlier with the circuit depth. Their fitted values averaged over the considered $\kappa_{\mathrm{wg}} / \kappa$ ratios are summarized in Table III.

\section{PIC interconnect}

In contrast with the GLM scheme, the teleportation scheme requires greater connectivity in the qRAM. Each node is not only connected to two children nodes in the next layer, but also to the rest of the nodes in the same layer. Here, we detail its PIC construct. Importantly, as shown in Fig. 15(a), the architecture requires interconnect layers interspersed between the binary tree layers. Additionally, a photon-detection system resides between each neighboring pair. Assuming that the single photons are propagating in one direction, i.e., incoming from the right of each layer, the detector would register photons after they interact with the cavities to its right.

Within each interconnect layer, MZI switches are classically controlled to enable routing of the single photons to individual cavities. The cavity depicted in Fig. 15(b) is the same construct shown in Fig. 10. To entangle two neighboring nodes, each tree node first operates in the setting mode. A single photon reflects off the first cavity and is directed to the second cavity via a circulator. After entering the second node through the MZI and reflecting off the cavity, the photon is again routed to an MZI switch via a circulator-except that now, the switch directs the photon to the detector for heralding a Bell-state creation. We stress

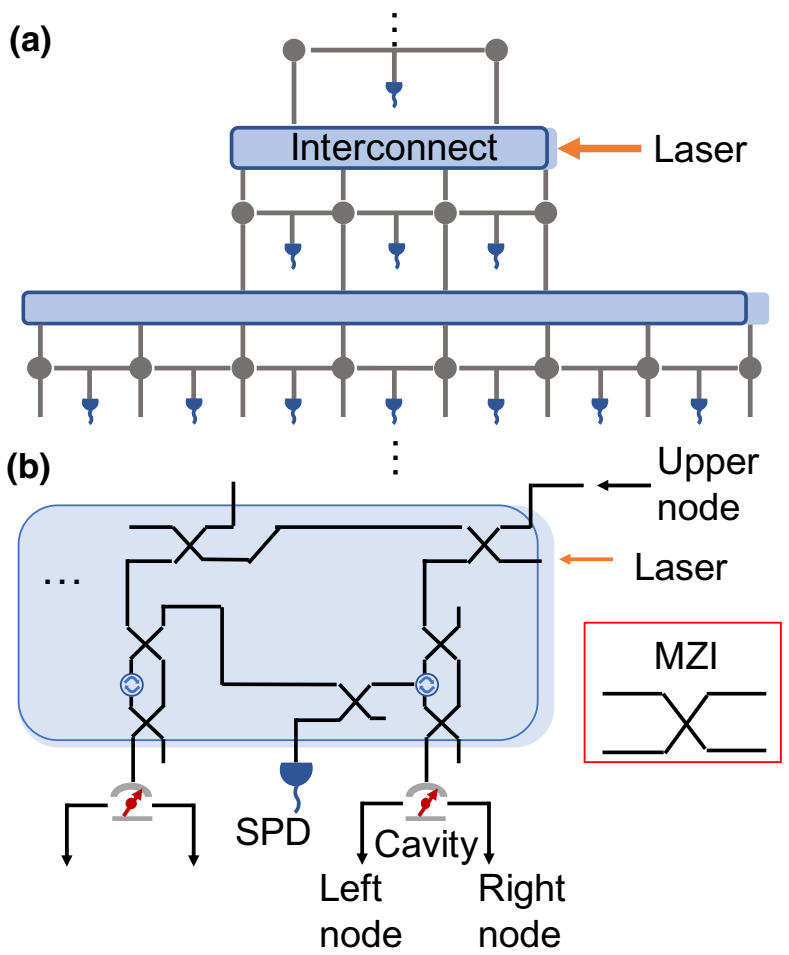

FIG. 15. The proposed PIC architecture for the teleportation scheme. (a) The qRAM binary tree contains interspersed interconnect layers that enable intralayer connectivity. (b) Within each interconnect layer, a network of MZIs is classically controlled to direct the single photons to either the subsequent cavity or the detection system for heralding during GHZ-state creation. It is then switched to a transparent state during the data retrieval step.

here that the interconnect layer enables beyond-nearestneighbor connection. Therefore, given prior knowledge of the query addresses, the architecture provides the ability to only entangle the necessary nodes and reduces state infidelity.

After the addresses are teleported from the QC to the qRAM, the cavity nodes are changed to the routing mode to direct the bus qubit to the memory layer at the bottom of the binary tree. In this step, the interconnect layer is essentially transparent by having the photon bypassing the circulators.

\section{Infidelity from decoherence}

We define the query fidelity as the fidelity of the prepared tree state. To calculate the infidelity caused by decoherence for each layer, we take the decoherence rate $\gamma_{d}$ to be proportional to $N t_{e \rightarrow n} / T_{2, n / e}$, where $N$ is the number of nodes, $T_{2, n / e}$ is the coherence time for the nuclear (electron) [subscript $n(e)]$ spin, and $t_{e \rightarrow n}$ is the approximate entanglement time. We assume perfect single qubit rotations, readout, and setting fidelity by optimally balancing losses (see Sec. II A). We only consider the effect of 


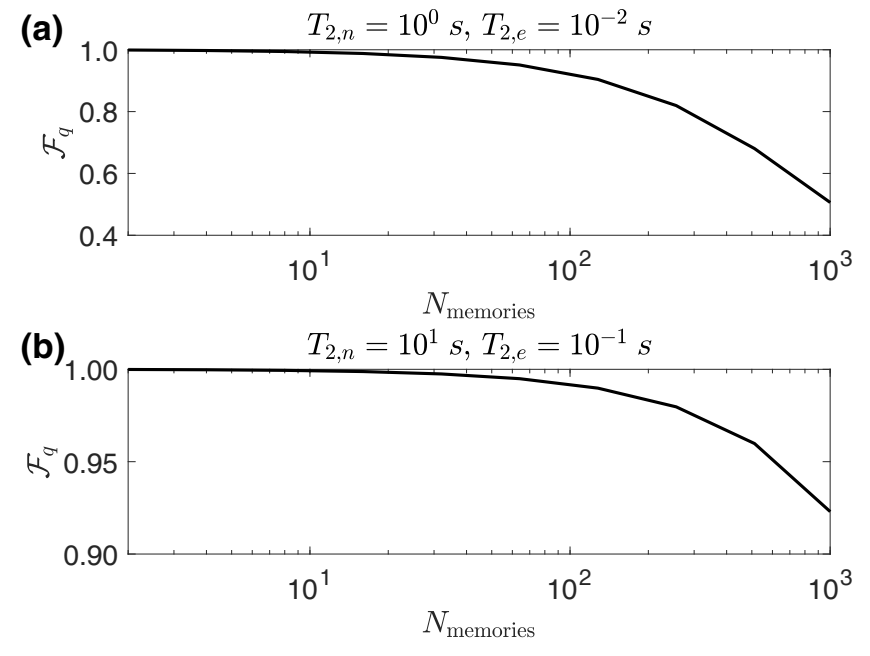

FIG. 16. The query fidelity as a function of the qRAM size. The nuclear and electron spin coherence times are, respectively, assumed to be: (a) $T_{2, n}=10^{0} \mathrm{~s}$ and $T_{2, e}=10^{-2} \mathrm{~s}$, (b) $T_{2, n}=$ $10^{1} \mathrm{~s}$ and $T_{2, e}=10^{-1} \mathrm{~s}$.

decoherence caused by continuous dephasing and neglect other physical errors such as imperfect nuclear-electron spin interaction. In Fig. 16(a), at $N_{\text {memories }}=10^{3}$, the query fidelity drops to near $\mathcal{F}_{q}=0.5$, suggesting that the prepared tree is no better than a maximally mixed state. Improvements on the nuclear and electron spin coherence times by an order of magnitude can increase the fidelity to $\mathcal{F}_{q}>0.9$, as shown in Fig. 16(b).

[1] V. Giovannetti, S. Lloyd, and L. Maccone, Quantum Random Access Memory, Phys. Rev. Lett. 100, 160501 (2008).

[2] V. Giovannetti, S. Lloyd, and L. Maccone, Architectures for a quantum random access memory, Phys. Rev. A 78, 052310 (2008).

[3] J. Biamonte, P. Wittek, N. Pancotti, P. Rebentrost, N. Wiebe, and S. Lloyd, Quantum machine learning, Nature 549, 195 (2017).

[4] A. W. Harrow, A. Hassidim, and S. Lloyd, Quantum Algorithm for Linear Systems of Equations, Phys. Rev. Lett. 103, 150502 (2009).

[5] B. T. Kiani, A. Villanyi, and S. Lloyd, Quantum medical imaging algorithms, ArXiv:2004.02036 (2020).

[6] L. K. Grover, in Proceedings, 28th Annual ACM Symposium on the Theory of Computing (ACM Press, New York, NY, USA, 1996).

[7] F.-Y. Hong, Y. Xiang, Z.-Y. Zhu, L.-Z. Jiang, and L.-N. Wu, Robust quantum random access memory, Phys. Rev. A 86, 010306 (2012).

[8] E. S. Moiseev and S. A. Moiseev, Time-bin quantum ram, J. Mod. Opt. 63, 2081 (2016).

[9] C. T. Hann, C.-L. Zou, Y. Zhang, Y. Chu, R. J. Schoelkopf, S. M. Girvin, and L. Jiang, Hardware-Efficient Quantum Random Access Memory with Hybrid Quantum Acoustic Systems, Phys. Rev. Lett. 123, 250501 (2019).
[10] H.-H. Lu, J. M. Lukens, N. A. Peters, O. D. Odele, D. E. Leaird, A. M. Weiner, and P. Lougovski, Electro-Optic Frequency Beam Splitters and Tritters for High-Fidelity Photonic Quantum Information Processing, Phys. Rev. Lett. 120, 030502 (2018).

[11] C. Joshi, A. Farsi, A. Dutt, B. Y. Kim, X. Ji, Y. Zhao, A. M. Bishop, M. Lipson, and A. L. Gaeta, Frequency-Domain Quantum Interference with Correlated Photons from an Integrated Microresonator, Phys. Rev. Lett. 124, 143601 (2020)

[12] H.-H. Lu, E. M. Simmerman, P. Lougovski, A. M. Weiner, and J. M. Lukens, Fully arbitrary control of frequency-bin qubits, ArXiv:2008.07444 (2020).

[13] C. T. Nguyen, D. D. Sukachev, M. K. Bhaskar, B. Machielse, D. S. Levonian, E. N. Knall, P. Stroganov, C. Chia, M. J. Burek, R. Riedinger, H. Park, M. Loncar, and M. D. Lukin, An integrated nanophotonic quantum register based on silicon-vacancy spins in diamond, Phys. Rev. B 100, 165428 (2019).

[14] M. K. Bhaskar, R. Riedinger, B. Machielse, D. S. Levonian, C. T. Nguyen, E. N. Knall, H. Park, D. Englund, M. Loncar, D. D. Sukachev, and M. D. Lukin, Experimental demonstration of memory-enhanced quantum communication, Nature 580, 60 (2020).

[15] N. C. Harris, G. R. Steinbrecher, M. Prabhu, Y. Lahini, J. Mower, D. Bunandar, C. Chen, F. N. C. Wong, T. BaehrJones, M. Hochberg, S. Lloyd, and D. Englund, Quantum transport simulations in a programmable nanophotonic processor, Nat. Photonics 11, 447 (2017).

[16] L.-M. Duan and H. J. Kimble, Scalable Photonic Quantum Computation through Cavity-Assisted Interactions, Phys. Rev. Lett. 92, 127902 (2004).

[17] D. D. Sukachev, A. Sipahigil, C. T. Nguyen, M. K. Bhaskar, R. E. Evans, F. Jelezko, and M. D. Lukin, Silicon-Vacancy Spin Qubit in Diamond: A Quantum Memory Exceeding 10 ms with Single-Shot State Readout, Phys. Rev. Lett. 119, 223602 (2017).

[18] M. A. Nielsen and I. L. Chuang, Quantum Computation and Quantum Information (Cambridge University Press, Cambridge, UK, 2010). 10th ed..

[19] M. D. Bowdrey, D. K. L. Oi, A. J. Short, K. Banaszek, and J. A. Jones, Fidelity of single qubit maps, Phys. Lett. A 294, 258 (2002).

[20] C. Y. Hu, A. Young, J. L. O’Brien, W. J. Munro, and J. G. Rarity, Giant optical Faraday rotation induced by a singleelectron spin in a quantum dot: Applications to entangling remote spins via a single photon, Phys. Rev. B 78, 085307 (2008)

[21] T. G. Tiecke, J. D. Thompson, N. P. de Leon, L. R. Liu, V. Vuletic, and M. D. Lukin, Nanophotonic quantum phase switch with a single atom, Nature 508, 241 (2014).

[22] Connor T. Hann, Gideon Lee, S. M. Girvin, and Liang Jiang, Resilience of quantum random access memory to generic noise, PRX Quantum 2, 020311 (2021).

[23] X. Rong, J. Geng, F. Shi, Y. Liu, K. Xu, W. Ma, F. Kong, Z. Jiang, Y. Wu, and J. Du, Experimental fault-tolerant universal quantum gates with solid-state spins under ambient conditions, Nat. Commun. 6, 8748 (2015).

[24] N. Kalb, A. A. Reiserer, P. C. Humphreys, J. J. W. Bakermans, S. J. Kamerling, N. H. Nickerson, S. C. Benjamin, 
D. J. Twitchen, M. Markham, and R. Hanson, Entanglement distillation between solid-state quantum network nodes, Science 356, 928 (2017).

[25] M. H. Abobeih, J. Cramer, M. A. Bakker, N. Kalb, M. Markham, D. J. Twitchen, and T. H. Taminiau, Onesecond coherence for a single electron spin coupled to a multi-qubit nuclear-spin environment, Nat. Commun. 9, 2552 (2018).

[26] K. D. Jahnke, B. Naydenov, T. Teraji, S. Koizumi, T. Umeda, J. Isoya, and F. Jelezko, Long coherence time of spin qubits in $12^{\mathrm{C}}$ enriched polycrystalline chemical vapor deposition diamond, Appl. Phys. Lett. 101, 021405 (2012).

[27] N. H. Nickerson, J. F. Fitzsimons, and S. C. Benjamin, Freely Scalable Quantum Technologies Using Cells of 5to-50 Qubits with Very Lossy and Noisy Photonic Links, Phys. Rev. X 4, 041041 (2014).

[28] H. Choi, M. Pant, S. Guha, and D. Englund, Percolationbased architecture for cluster state creation using photonmediated entanglement between atomic memories, npj Quantum Inf. 5, 104 (2019).

[29] N. H. Wan, T.-J. Lu, K. C. Chen, M. P. Walsh, M. E. Trusheim, L. De Santis, E. A. Bersin, I. B. Harris, S. L. Mouradian, I. R. Christen, E. S. Bielejec, and D. Englund, Large-scale integration of near-indistinguishable artificial atoms in hybrid photonic circuits, Nature 583, 226 (2020).

[30] G. Thiering and A. Gali, Ab Initio Magneto-Optical Spectrum of Group-IV Vacancy Color Centers in Diamond, Phys. Rev. X 8, 021063 (2018).

[31] S. Sangtawesin, B. L. Dwyer, S. Srinivasan, J. J. Allred, L. V. H. Rodgers, K. De Greve, A. Stacey, N. Dontschuk, K. M. O’Donnell, D. Hu, D. A. Evans, C. Jaye, D. A. Fischer, M. L. Markham, D. J. Twitchen, H. Park, M. D. Lukin, and N. P. de Leon, Origins of Diamond Surface Noise Probed by Correlating Single-Spin Measurements with Surface Spectroscopy, Phys. Rev. X 9, 031052 (2019).

[32] S. Meesala, Y.-I. Sohn, B. Pingault, L. Shao, H. A. Atikian, J. Holzgrafe, M. Gundogan, C. Stavrakas, A. Sipahigil, C. Chia, R. Evans, M. J. Burek, M. Zhang, L. Wu, J. L. Pacheco, J. Abraham, E. Bielejec, M. D. Lukin, M. Atature, and M. Lončar, Strain engineering of the silicon-vacancy center in diamond, Phys. Rev. B 97, 205444 (2018).
[33] Q. Quan, P. B. Deotare, and M. Loncar, Photonic crystal nanobeam cavity strongly coupled to the feeding waveguide, Appl. Phys. Lett. 96, 203102 (2010).

[34] A. Alajlan, I. Cojocaru, and A. V. Akimov, Compact design of a gallium phosphide nanobeam cavity for coupling to diamond germanium-vacancy centers, Opt. Mater. Express 9, 1678 (2019).

[35] J. P. Vasco, V. Savona, and D. Gerace, Optimized design of a silica encapsulated photonic crystal nanobeam cavity for integrated silicon-based nonlinear and quantum photonics, ArXiv:1910.10647 (2019).

[36] M. Zhang, C. Wang, R. Cheng, A. Shams-Ansari, and M. Loncar, Monolithic ultra-high- $Q$ lithium niobate microring resonator, Optica 4, 1536 (2017).

[37] B. Desiatov, A. Shams-Ansari, M. Zhang, C. Wang, and M. Loncar, Ultra-low-loss integrated visible photonics using thin-film lithium niobate, Optica 6, 380 (2019).

[38] S. Sun, H. Kim, G. S. Solomon, and E. Waks, A quantum phase switch between a single solid-state spin and a photon, Nat. Nanotechnol. 11, 539 (2016).

[39] J. M. Kindem, A. Ruskuc, J. G. Bartholomew, J. Rochman, Y. Q. Huan, and A. Faraon, Control and single-shot readout of an ion embedded in a nanophotonic cavity, Nature 580, 2 (2020).

[40] I. Pogorelov, T. Feldker, C. D. Marciniak, L. Postler, G. Jacob, O. Kriegelsteiner, V. Podlesnic, M. Meth, V. Negnevitsky, M. Stadler, B. Hofer, C. Wachter, K. Lakhmanskiy, R. Blatt, P. Schindler, and T. Monz, A compact ion-trap quantum computing demonstrator, ArXiv:2101.11390 (2021).

[41] A. Omran, H. Levine, A. Keesling, G. Semeghini, T. T. Wang, S. Ebadi, H. Bernien, A. S. Zibrov, H. Pichler, S. Choi, J. Cui, M. Rossignolo, P. Rembold, S. Montangero, T. Calarco, M. Endres, M. Greiner, V. Vuletic, and M. D. Lukin, Generation and manipulation of Schrödinger cat states in Rydberg atom arrays, Science 365, 570 (2019).

[42] G. Barbarossa, A. M. Matteo, and M. N. Armenise, Theoretical analysis of triple-coupler ring-based optical guided-wave resonator, J. Lightwave Technol. 13, 148 (1995). 